\title{
Star-forming dwarf galaxies in the Virgo cluster: the link between molecular gas, atomic gas, and dust ${ }^{\star, \star \star}$
}

\author{
M. Grossi ${ }^{1}$, E. Corbelli ${ }^{2}$, L. Bizzocchi ${ }^{3}$, C. Giovanardi ${ }^{2}$, D. Bomans ${ }^{4}$, B. Coelho ${ }^{1}$, I. De Looze ${ }^{5,6}$, T. S. Gonçalves ${ }^{1}$, \\ L. K. Hunt ${ }^{2}$, E. Leonardo ${ }^{7,8}$, S. Madden ${ }^{9}$, K. Menéndez-Delmestre ${ }^{1}$, C. Pappalardo ${ }^{7,8}$, and L. Riguccini ${ }^{1}$ \\ ${ }^{1}$ Observatório do Valongo, Universidade Federal do Rio de Janeiro, Ladeira Pedro Antônio 43, Rio de Janeiro, Brazil \\ e-mail: grossi@astro.ufrj.br \\ 2 INAF-Osservatorio Astrofisico di Arcetri, Largo Enrico Fermi 5, 50125 Firenze, Italy \\ 3 Center for Astrochemical Studies, Max-Planck-Institut für extraterrestrische Physik (MPE), Giessenbachstraße 1, 85748 Garching, \\ Germany \\ 4 Astronomical Institute of the Ruhr-University Bochum (AIRUB), Universitätstr. 150, 44801 Bochum, Germany \\ 5 Sterrenkundig Observatorium, Universiteit Gent, Krijgslaan 281, 9000 Gent, Belgium \\ 6 Department of Physics and Astronomy, University College London, Gower Street, London WC1E 6BT, UK \\ 7 Instituto de Astrofísica e Ciências do Espaço, Universidade de Lisboa, OAL, Tapada da Ajuda, 1349-018 Lisboa, Portugal \\ 8 Departamento de Física, Faculdade de Ciências, Universidade de Lisboa, Campo Grande, 1749-016 Lisboa, Portugal \\ 9 Laboratoire AIM, CEA/DSM - CNRS - Université Paris Diderot, IRFU/Service d'Astrophysique, CEA Saclay, \\ 91191 Gif-sur-Yvette, France
}

Received 13 January 2016 / Accepted 19 February 2016

\begin{abstract}
We present ${ }^{12} \mathrm{CO}(1-0)$ and ${ }^{12} \mathrm{CO}(2-1)$ observations of a sample of 20 star-forming dwarfs selected from the Herschel Virgo Cluster Survey, with oxygen abundances ranging from $12+\log (\mathrm{O} / \mathrm{H}) \sim 8.1$ to 8.8. $\mathrm{CO}$ emission is observed in ten galaxies and marginally detected in another one. CO fluxes correlate with the FIR $250 \mu \mathrm{m}$ emission, and the dwarfs follow the same linear relation that holds for more massive spiral galaxies extended to a wider dynamical range. We compare different methods to estimate $\mathrm{H}_{2}$ molecular masses, namely a metallicity-dependent $\mathrm{CO}$-to- $\mathrm{H}_{2}$ conversion factor and one dependent on $H$-band luminosity. The molecular-to-stellar mass ratio remains nearly constant at stellar masses $\lesssim 10^{9} M_{\odot}$, contrary to the atomic hydrogen fraction, $M_{\mathrm{HI}} / M_{*}$, which increases inversely with $M_{*}$. The flattening of the $M_{\mathrm{H}_{2}} / M_{*}$ ratio at low stellar masses does not seem to be related to the effects of the cluster environment because it occurs for both HI-deficient and HI-normal dwarfs. The molecular-to-atomic ratio is more tightly correlated with stellar surface density than metallicity, confirming that the interstellar gas pressure plays a key role in determining the balance between the two gaseous components of the interstellar medium. Virgo dwarfs follow the same linear trend between molecular gas mass and star formation rate as more massive spirals, but gas depletion timescales, $\tau_{\text {dep }}$, are not constant and range between 100 Myr and 6 Gyr. The interaction with the Virgo cluster environment is removing the atomic gas and dust components of the dwarfs, but the molecular gas appears to be less affected at the current stage of evolution within the cluster. However, the correlation between HI deficiency and the molecular gas depletion time suggests that the lack of gas replenishment from the outer regions of the disc is lowering the star formation activity.
\end{abstract}

Key words. galaxies: dwarf - galaxies: ISM - galaxies: clusters: individual: Virgo - galaxies: evolution

\section{Introduction}

Star-forming dwarf (SFD) galaxies are rich in atomic hydrogen (HI), but despite their star-formation activity, the detection of molecular gas in these systems is challenging. The lack of low-energy transitions in the molecular hydrogen molecule $\left(\mathrm{H}_{2}\right)$, which is due to the absence of a permanent dipole moment, implies that the $\mathrm{H}_{2}$ content has to be inferred from the emission of carbon monoxide (CO; Young \& Scoville 1991). While $\mathrm{H}_{2}$ is self-shielded from the UV radiation, dust is primarily responsible for preventing $\mathrm{CO}$ photodissociation (Wolfire et al. 2010), therefore only dense CO clumps can survive in dust-poor,

\footnotetext{
* Based on observations carried out with the IRAM 30-m telescope. IRAM is supported by INSU/CNRS (France), MPG (Germany) and IGN (Spain).

$\star \star$ The reduced CO spectra are only available at the CDS via

anonymous ftp to cdsarc.u-strasbg. fr (130.79.128.5) or via

http://cdsarc.u-strasbg.fr/viz-bin/qcat?J/A+A/590/A27
}

low-metallicity systems (Lequeux et al. 1994; Israel et al. 2003; Leroy et al. 2009a). It is unclear to which extent a lack of CO emission means a correspondingly low $\mathrm{H}_{2}$ content because a significant $\mathrm{H}_{2}$ mass may lie outside the $\mathrm{CO}$ region, in the outer parts of the molecular cloud where carbon is in the atomic phase (Poglitsch et al. 1995; Madden et al. 1997). Indeed, carbon monoxide is often undetected in very metal-poor lowmass galaxies with a threshold abundance usually observed at $Z \simeq 0.2 Z_{\odot}$ (Taylor et al. 1998; Leroy et al. 2005; Schruba et al. 2012), and the CO detection rate is usually higher in more massive Magellanic-type dwarf galaxies than in irregulars and blue compact dwarfs (BCDs; Albrecht et al. 2004).

The conversion between $\mathrm{CO}$ intensity and $\mathrm{H}_{2}$ abundance has been studied for more than two decades (for a review, see Bolatto et al. 2013). The existence of a standard CO-to- $\mathrm{H}_{2}$ mass conversion factor, $X_{\mathrm{CO}}$, and its dependence on the physical conditions of the interstellar medium (ISM) has been highly debated (Israel 1997; Leroy et al. 2011): metallicity, ionising 
stellar radiation field strength and the density of the gas are among the main parameters that can affect the value of the $X_{\text {CO }}$ factor in galaxies (Wilson 1995; Israel 1997; Boselli et al. 2002; Tacconi et al. 2008; Leroy et al. 2011; Shetty et al. 2011; Schruba et al. 2012; Sandstrom et al. 2013)

Because large $\mathrm{H}_{2}$ envelopes may be missed in the census of the ISM gas components, cold dust $(T<30 \mathrm{~K})$ has recently been used as an indirect tracer of the total gas content (e.g. Bot et al. 2007; Leroy et al. 2011; Groves et al. 2015). Dust is observed to be well mixed with gas (e.g. Bohlin et al. 1978; Boulanger et al. 1996) and can be mapped by its emission at far-infrared (FIR) wavelengths. Corbelli et al. (2012) showed that there is a linear relation between cold dust emission and $\mathrm{CO}$ brightness in spiral galaxies down to $S_{\mathrm{CO}}=200 \mathrm{~K} \mathrm{~km} \mathrm{~s}^{-1}$ (or $M_{\mathrm{H}_{2}}=2 \times 10^{8} M_{\odot}$, assuming a galactic conversion factor). However, no metallicity dependence was investigated in their sample because of the lack of systems with abundances below the galactic value.

In this paper we present and analyse ${ }^{12} \mathrm{CO}(1-0)$ and ${ }^{12} \mathrm{CO}(2-1)$ observations of SFDs in the Virgo cluster with the Institut de Radio Astronomie Millimétrique (IRAM) 30-m telescope. These galaxies were detected at FIR/submillimetre (submm) wavelengths with Herschel (Grossi et al. 2015), as part of the Herschel Virgo Cluster survey (HeViCS; Davies et al. 2012; Auld et al. 2013). Oxygen abundances span a range of solar and sub-solar values with $12+\log (\mathrm{O} / \mathrm{H})$ between 8.1 and $8.8 \mathrm{dex}^{1}$.

Such a sample of dwarf galaxies is ideal to extend the dynamical range of the cold dust-CO emission correlation established for Virgo spiral galaxies down to lower stellar masses and sub-solar metallicities. Moreover, using the $\mathrm{CO}$ lines to infer the molecular hydrogen masses, we aim to shed light on the ISM of dwarf galaxies and on the relation between dust, atomic and molecular gas components, and on possible environmental dependencies.

HeViCS observations of higher mass galaxies combined with $\mathrm{HI}$ and $\mathrm{CO}$ data have shown that dust removal in Virgo HI-deficient spiral galaxies is lower than the amount of stripped neutral hydrogen, and that the molecular component, well confined into the deep potential well, is affected even less by the dense environment (Cortese et al. 2010, 2011; Corbelli et al. 2012). Boselli et al. (2014b) showed that galaxies in the core of the Virgo cluster are modestly deficient in molecular gas, in agreement with previous results based on a smaller sample of galaxies (Boselli \& Gavazzi 2006; Fumagalli et al. 2009). Highly HI-deficient Virgo SFD galaxies are mostly characterised by reduced star formation activity and lower dust fractions (Grossi et al. 2015), therefore we here wish to investigate how the molecular component of dwarfs can be affected by the cluster environment.

The paper is organised as follows. In Sect. 2 we describe IRAM observations and data reduction. The analysis of the $\mathrm{CO}$ observations is discussed in Sect. 3. The properties of the detected and non-detected galaxies are compared in Sect. 4. In Sect. 5 we analyse the correlation between FIR flux densities and integrated CO-line intensities. We estimate the molecular masses in Sect. 6. The molecular hydrogen properties of the Virgo SFDs are presented and discussed in Sect. 7 and include scaling relations of the $\mathrm{H}_{2}$ component (Sect. 7.1); the molecular-to-atomic gas mass ratios (Sect. 7.2), dust-to-gas ratios (Sect. 7.3), the molecular, atomic, and total gas mass starformation laws (Sect. 7.4), and the environmental effects on the

\footnotetext{
1 Throughout this work we assume a solar oxygen abundance of $12+\log (\mathrm{O} / \mathrm{H})=8.69$ (Asplund et al. 2009).
}

molecular gas (Sect. 7.5). Finally, in Sect. 8 we summarise our results and conclusions.

\section{Sample selection, observations, and data reduction}

\subsection{Sample selection}

The sample of SFD galaxies observed at the IRAM 30-m telescope was defined according to the following criteria: i) morphological classification as blue compact dwarfs (BCD) or Magellanic spirals and irregulars (Sm, Im), according to the Virgo Cluster Catalog (VCC; Binggeli et al. 1985) and GOLDMine (Gavazzi et al. 2003, 2014) and ii) detectable FIR emission in four Herschel bands $(100,160,250$, and $350 \mu \mathrm{m})$ above $5 \sigma$. These criteria produced a selection of 23 galaxies. We added the galaxy VCC172 to the sample because of its proximity to VCC144 both in projected distance $\left(\sim 1^{\circ}\right)$ and velocity $(\Delta V \sim$ $\left.150 \mathrm{~km} \mathrm{~s}^{-1}\right)$, although its detection at $350 \mu \mathrm{m}$ is slightly below $5 \sigma$. Only 20 targets were observed because of bad weather conditions during the observing runs ${ }^{2}$. Table 1 displays the main properties of the observed sample as derived in Grossi et al. (2015). In Fig. 1 we show the range of stellar and HI masses, star formation rates (SFRs), metallicities, HI-to-stellar mass ratios, and the projected distance from either M87 or M49, the elliptical galaxies at the core of the two main substructures in Virgo, cluster A and cluster B (Binggeli et al. 1985). Filled histograms correspond to the detected galaxies in at least one $\mathrm{CO}$ line. We briefly summarise below the methods followed to estimate these parameters, but we refer to Grossi et al. (2015) for further details.

Stellar masses were calculated following the approach of Wen et al. (2013), which is based on $3.4 \mu \mathrm{m}$ photometry with the WISE telescope (Wright et al. 2010). Atomic hydrogen (HI) masses were derived from the Arecibo Legacy Fast ALFA (ALFALFA) blind HI survey (Giovanelli et al. 2005), using the $\alpha .40$ catalogue release (Haynes et al. 2011). The HI deficiency parameter, $D e f_{\mathrm{HI}^{3}}{ }^{3}$ (Haynes \& Giovanelli 1984), was estimated following Gavazzi et al. (2013). Star-formation rates were calculated from $\mathrm{H} \alpha$ photometry obtained from the GOLDMine data base (Gavazzi et al. 2003, 2014). Mid-infrared photometry at $22 \mu \mathrm{m}$ from WISE archive images was measured to take into account the contribution of dust-obscured star formation using the relation of Wen et al. (2014). For those galaxies without a WISE detection, we calculated the SFR from the $\mathrm{H} \alpha$ fluxes alone, using Kennicutt (1998a) for a Kroupa (2001) IMF, after correcting the $\mathrm{H} \alpha$ fluxes for internal extinction from the Balmer decrement measured in the Sloan Digital Sky Survey (SDSS) spectra (York et al. 2000). Oxygen abundances were derived from the SDSS based with the method of Hughes et al. (2013). The method combines five metallicity calibrations and converts them into a base metallicity, that is, the O3N2 $=[\mathrm{OIII}] \lambda 5007 /[\mathrm{NII}] \lambda 6584$ index of Pettini \& Pagel (2004). However, because the [OII] $\lambda 3727$ line is out of the measured wavelength range of the SDSS at the distance of Virgo, the only applicable calibrations for our sample were those based on the N2 $=[\mathrm{NII}] \lambda 6584 / \mathrm{H} \alpha$ and O3N2 indices of Pettini \& Pagel (2004). The oxygen abundance of VCC1686 was derived from

2 Of the four non-observed galaxies, VCC1554 was detected at $3 \mathrm{~mm}$ by Boselli et al. (2014a) as part of the CO follow-up observations of the Herschel Reference Survey (Boselli et al. 2010).

3 The HI deficiency is defined as the logarithmic difference between the HI mass of a reference sample of isolated galaxies for a given morphological type and the observed HI mass, $D e f_{\mathrm{HI}}=\log M_{\mathrm{HI}}^{\mathrm{ref}}-\log M_{\mathrm{HI}}^{\mathrm{obs}}$. 

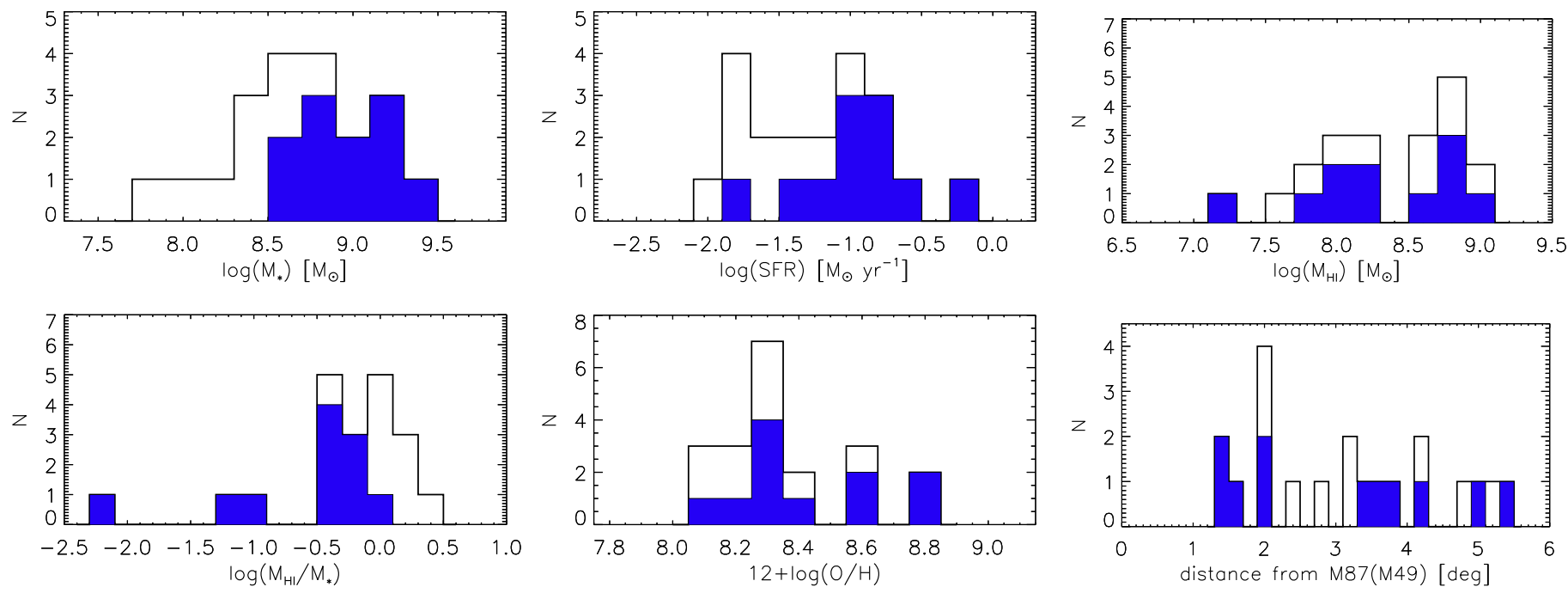

Fig. 1. Main properties of the Virgo SFDs observed in this work: stellar mass, SFR, HI mass, HI-to-stellar mass ratio, metallicity, and projected distance from either M87 or M49. Filled histograms show the CO-detected galaxies.

Table 1. Main properties of the Virgo SFDs.

\begin{tabular}{|c|c|c|c|c|c|c|c|c|c|c|}
\hline ID & $\begin{array}{c}\log \left(M_{\star}\right) \\
{\left[M_{\odot}\right]} \\
\end{array}$ & $\begin{array}{c}\log \left(M_{\mathrm{HI}}\right) \\
{\left[M_{\odot}\right]} \\
\end{array}$ & $\begin{array}{c}\log \left(M_{\mathrm{d}}\right) \\
{\left[M_{\odot}\right]}\end{array}$ & $\begin{array}{l}\log (S F R) \\
{\left[M_{\odot} \mathrm{yr}^{-1}\right]}\end{array}$ & l) & $\mathrm{HI}$ & $\begin{array}{c}\Delta V_{\mathrm{HI}} \\
{\left[\mathrm{km} \mathrm{s}^{-1}\right]}\end{array}$ & $\begin{array}{c}D \\
{[\mathrm{Mpc}]}\end{array}$ & $\begin{array}{l}a_{25} \\
\left({ }^{\prime \prime}\right) \\
\end{array}$ & $b_{25}$ \\
\hline & $8.95 \pm 0.04$ & $74 \pm 0.01$ & $6+0+006$ & $05 \pm 0.08$ & to & & 186 & 32.0 & 61.8 & 13.2 \\
\hline VCC87 & $39 \pm 0.04$ & $51 \pm$ & & $.62 \pm 0.07$ & $25 \pm 0.10$ & 17 & (1) & 17.0 & 87.0 & 43.2 \\
\hline VCC135 & $9.44 \pm 0.04$ & $.19 \pm 0.08$ & $6.19_{-0.06}^{+0.06}$ & $-1.03 \pm 0.08$ & $65 \pm 0.10$ & 1.73 & $123^{a}$ & & 69.6 & 34.2 \\
\hline VCC144 & $8.81 \pm$ & $8.76 \pm$ & & $-0.27=$ & & & & & 37.8 & 19.2 \\
\hline VCC172 & $8.88 \pm($ & $.95 \pm 0.01$ & & $.45 \pm$ & 10 & & 126 & & 5.6 & 33.6 \\
\hline VCC213 & $8.89 \pm$ & $7.84 \pm 0.03$ & & $-1.20 \pm 0.06$ & $.77 \pm 0.12$ & 0.57 & 122 & 17.0 & 55.8 & 42.6 \\
\hline VCC324 & $8.72 \pm 0.04$ & $8.23 \pm 0.01$ & & $-0.75 \pm 0.07$ & & (a) & $45^{a}$ & 17.0 & 81.0 & 69.0 \\
\hline vCC334 & 04 & 01 & 4.9 & $-177+$ & & & & 17.0 & 6 & 30.6 \\
\hline VCC340 & 04 & $8.89 \pm 0.01$ & & $-0.84 \pm$ & & & & 32 . & 66.0 & 25.8 \\
\hline VCC562 & $7.76=$ & $7.74 \pm$ & & & & 0 & 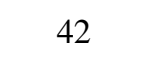 & 17.0 & 37.8 & 29.4 \\
\hline VCC693 & $832-4$ & $827+4$ & 5.5 & $-1.93=$ & & & & 17.0 & 69.6 & 60.0 \\
\hline VCC699 & $9.19 \pm 0.04$ & $8.94 \pm 0.01$ & & $-0.63 \pm 0.06$ & - & & & 23.0 & 117.0 & 82.8 \\
\hline VCC737 & & 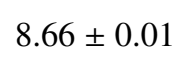 & & & & & 1 & 1 & 64.2 & 21.0 \\
\hline & & & & & & & 39 & 17.0 & 50.4 & 17.4 \\
\hline VCC1437 & $8.52 \pm 0.04$ & $8.03 \pm 0.02$ & $5.23^{+}$ & $-1.78 \pm 0.17$ & & & 57 & 17.0 & 35.4 & 27.0 \\
\hline VCC 1575 & 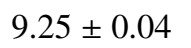 & $7.97 \pm 0.02$ & & $-0.90 \pm 0.10$ & & & & 17.0 & 120.0 & 84.6 \\
\hline $\mathrm{VCC}$ & & & & & & & 105 & 17.0 & 167.4 & 102.6 \\
\hline VCC1699 & $8.57 \pm 0.04$ & $8.77 \pm 0.01$ & & $-1.12 \pm 0.08$ & $8.07 \pm 0.12$ & -0.06 & 86 & 17.0 & 93.0 & 49.8 \\
\hline VCC 1725 & $8.59 \pm 0.04$ & $8.21 \pm 0.01$ & 5.7 & $-1.36 \pm 0.07$ & $8.25 \pm 0.10$ & 0.50 & 76 & 17.0 & 93.0 & 58.2 \\
\hline VCC1791 & $52 \pm 0.04$ & $8.72 \pm 0.01$ & $5.71_{-0.06}^{+0.07}$ & $-1.08 \pm 0.05$ & $8.16 \pm 0.10$ & -0.11 & 93 & 17.0 & 77.4 & 38.4 \\
\hline
\end{tabular}

Notes. ${ }^{(a)}$ Springob et al. (2005); ${ }^{(b)}$ Metallicity derived from the mass-metallicity relation of Hughes et al. (2013).

the mass metallicity relation of Hughes et al. (2013) because of the lack of SDSS spectroscopical observations. Dust masses were calculated by fitting a modified black-body (MBB) function to the FIR spectral energy distribution (SED) between 100 and $350 \mu \mathrm{m}$ assuming a fixed emissivity index $\beta=1.5$ and $\kappa_{0}=3.4 \mathrm{~cm}^{2} \mathrm{~g}^{-1}$ at $\lambda=250 \mu \mathrm{m}$, following the prescription of Bianchi (2013). The emissivity index $\beta=1.5$ was found to best fit the FIR SEDs minimising the fraction of residuals in the four Herschel bands among a range of five adopted values (1.0, 1.2, 1.5, 1.8, 2.0; see Grossi et al. 2015). Isophotal major $\left(a_{25}\right)$ and minor diameters $\left(b_{25}\right)$ at the 25th $B$-magnitude $\operatorname{arcsec}^{-2}$ and distances to the galaxies were extracted from the GOLDMine data base.

\subsection{IRAM observations}

$\mathrm{CO}(1-0)$ and $\mathrm{CO}(2-1)$ observations with the IRAM 30-m telescope (Pico Veleta, Spain) were obtained over the course of three observing runs: 2011 December 9-10, 2012 May 6-7, and 
201229 June - 2 July. The telescope full width half maximum (FWHM) is $21^{\prime \prime} 3$ and 10.77 at $115.27 \mathrm{GHz}$ and $230.54 \mathrm{GHz}$, respectively. This corresponds to $\sim 1.8 \mathrm{kpc}$ and $\sim 0.9 \mathrm{kpc}$ at a distance of $17 \mathrm{Mpc}$. The $30-\mathrm{m}$ telescope beam size at $115 \mathrm{GHz}$ is comparable to the Herschel/SPIRE resolution at $250 \mu \mathrm{m}\left(18^{\prime \prime}\right)$.

Most of our targets were observed with a single pointing because their optical extension and the size of the FIR-emitting region are comparable to the IRAM beam at $115 \mathrm{GHz}$ (Fig. 2). However, to obtain a more complete coverage of the molecular gas distribution, four galaxies were observed with more than one pointing with a spacing of half the beam size at $115 \mathrm{GHz}$ (VCC213, VCC699, VCC1575, VCC1686; see Fig. 2). Total integration times per target varied between 10 and $130 \mathrm{~min}$ (see Table A.1).

Observations were performed in reasonable weather conditions $(\tau(230 \mathrm{GHz}) \sim 0.07-0.42)$ using the Eight MIxer Receiver $\left(E M I R^{4}\right)$ in bands E090 and E230 to simultaneously detect $\mathrm{CO}(1-0)$ and $\mathrm{CO}(2-1)$ lines. However, during the third run, we used only the E090 band, hence for seven dwarf galaxies we only have $\mathrm{CO}(1-0)$ line observations. To increase the redundancy, Fast Fourier Transform Spectrometer (FTS) and WIdeband Line Multiple Autocorrelator (WILMA) were used in parallel to sample the data. FTS provides a bandwidth of $8 \mathrm{GHz}$ in each of the two orthogonal linear polarisations and a channel resolution of $200 \mathrm{kHz}$. The bandwidth of WILMA autocorrelator is $1 \mathrm{GHz}$ with a spectral resolution of $2 \mathrm{MHz}$. The wobblerswitching mode, with a $120^{\prime \prime}$ throw, was preferred given the small sizes of the targets. Pointing and focus were checked on nearby strong continuum sources and Mars and were monitored every two hours in stable conditions and every one hour during sunrise. The pointing accuracy was $\$ 3^{\prime \prime}$ and the system temperatures varied between $\sim 280$ and $\sim 700 \mathrm{~K}$ at $115 \mathrm{GHz}$ on the antenna temperatures $\left(T_{A}^{*}\right)$ scale. To convert $T_{A}^{*}$ into main beam temperatures $\left(T_{\mathrm{mb}}\right)$, we used $T_{\mathrm{mb}}=T_{\mathrm{a}}^{*} \times F_{\mathrm{eff}} / B_{\mathrm{eff}}$, with forward efficiencies $\left(F_{\text {eff }}\right)$ of 0.94 and 0.91 and main beam efficiencies $\left(B_{\text {eff }}\right)$ of 0.78 and 0.58 , at 115 and $230 \mathrm{GHz}$, respectively. Conversion between main beam temperature in Kelvin and flux in Jansky $\left(S_{v}\right)$ for a point-like source were made using the relation ${ }^{5}$

$G=\frac{S_{v}}{T_{\mathrm{mb}}}=8.16 \times 10^{-7}\left(\frac{v}{\mathrm{GHz}}\right)^{2}\left(\frac{\theta_{\mathrm{beam}}}{\operatorname{arcsec}}\right)^{2}[\mathrm{Jy} / \mathrm{K}]$,

where $\theta_{\text {beam }}$ is the FWHM of the beam at the observing frequency $v$, corresponding to a gain factor $G=4.94 \mathrm{Jy} / \mathrm{K}$.

\subsection{Data reduction}

The data were reduced with the GILDAS-CLASS $90^{6}$ software package (Pety 2005). Scans were averaged after fitting polynomial baselines, and they were boxcar-smoothed to a resolution of $\delta V=8.1 \mathrm{~km} \mathrm{~s}^{-1}$. The rms noise level of the final spectra ranges between $3 \mathrm{mK}$ and $11 \mathrm{mK}$ in both bands (Table 2).

Integrated intensities, $I_{\mathrm{CO}}$, were calculated in two ways: i) we fitted a Gauss function (single-peak profiles) or a linear combination of the first two Hermite functions (double-peak profiles; Saintonge 2007) to the spectra; and ii) we summed the spectra over the observed velocity range. Both methods give consistent values, which we display in Table 2 . We assumed that a galaxy was detected in one beam when the peak emission was above $3 \sigma$ or when the integrated emission over the defined velocity

\footnotetext{
4 http://www.iram.es/IRAMES/mainWiki/ EmirforAstronomers

5 Kramer 1997, IRAM report on Calibration of 30-m data

6 http://www.iram. fr/IRAMFR/GILDAS/
}

window was above $4 \sigma$. Of a total of 20 galaxies, we detected the $\mathrm{CO}(1-0)$ line in nine sources and the $\mathrm{CO}(2-1)$ line in ten targets. VCC10 and VCC144 were only detected at $230 \mathrm{GHz}$, while VCC1725 was marginally detected only at $115 \mathrm{GHz}$. Upper limits for the undetected galaxies were derived using

$I_{\mathrm{CO}}^{\mathrm{up}}=4 \sigma \sqrt{\Delta V_{\mathrm{up}} \delta V}$,

where $\delta V$ is the spectral resolution and $\Delta V_{\text {up }}$ is the assumed line width of the galaxy. When no CO lines were detected, $\Delta V_{\text {up }}$ corresponds to the HI line FWHM given in Table 1, and when only one line was detected, we used the measured FWHM to calculate the upper limit to the intensity of the other undetected line (e.g. VCC10, VCC144, VCC1575(e), VCC1686(c), VCC1725). The spectra of all the observed galaxies are displayed in Figs. B.1 and B.2.

In the rest of this work we present the results derived from the sum of the spectra over the observed velocity width. The errors on the integrated intensities are determined according to

$\Delta I_{\mathrm{CO}}=\sigma \sqrt{\Delta V_{\mathrm{CO}} \delta V_{\mathrm{CO}}}$

where $\sigma$ is the rms noise of the spectrum, $\Delta V_{\mathrm{CO}}$ the $\mathrm{CO}$ line width, and $\delta V_{\mathrm{CO}}$ the spectral resolution.

\section{Analysis of $\mathrm{CO}$ observations}

The majority of the galaxies in the sample were observed with a single pointing in their central position. Consequently, we may expect two types of situations when estimating the total $\mathrm{CO}$ brightness of the galaxy: 1) the telescope beam is smaller than the size of the CO-emitting area and corrections for incomplete coverage should be applied, or 2) the $\mathrm{CO}$ emission originates from a region smaller than the telescope beam, and we therefore need to determine its size and the corresponding correction factor after assuming the source geometry (usually a uniform disc or Gaussian.) We discuss the strategy to account for these effects below.

\subsection{Aperture correction for extended emission}

\subsubsection{Single-pointing observations}

To determine the aperture correction for an emitting region more extended than the beam, we followed two approaches: i) an empirical method calibrated on nearby mapped galaxies (Saintonge et al. 2011a); and ii) an analytic method that assumes an exponential radial profile for the distribution of the molecular gas (Lisenfeld et al. 2011).

The first method was proposed by Saintonge et al. (2011a), who simulated the effects of a single-beam observation on a sample of galaxies with IRAM 30-m telescope CO maps (Kuno et al. 2007), calculated the ratio between the total flux and the flux observed by a $\sim 22^{\prime \prime}$ beam, and obtained an empirical calibration. With this technique, the aperture correction, $f_{\text {ap }}$, defined as the ratio between the total (extrapolated) $\mathrm{CO}$ flux $\left(S_{\mathrm{CO}}^{\text {tot }}\right)$ and the flux in the central pointing $\left(S_{\mathrm{CO}}^{\text {central }}\right)$

$f_{\text {ap }}=S_{\mathrm{CO}}^{\text {tot }} / S_{\mathrm{CO}}^{\text {central }}$

is given by the following relation:

$S_{\mathrm{CO}}^{\text {tot }}=\frac{S_{\mathrm{CO}}^{\text {central }}}{1.094-0.008 D_{25}+2 \times 10^{-5} D_{25}^{2}}$, 
M. Grossi et al.: Star-forming dwarf galaxies in the Virgo cluster: the link between molecular gas, atomic gas, and dust
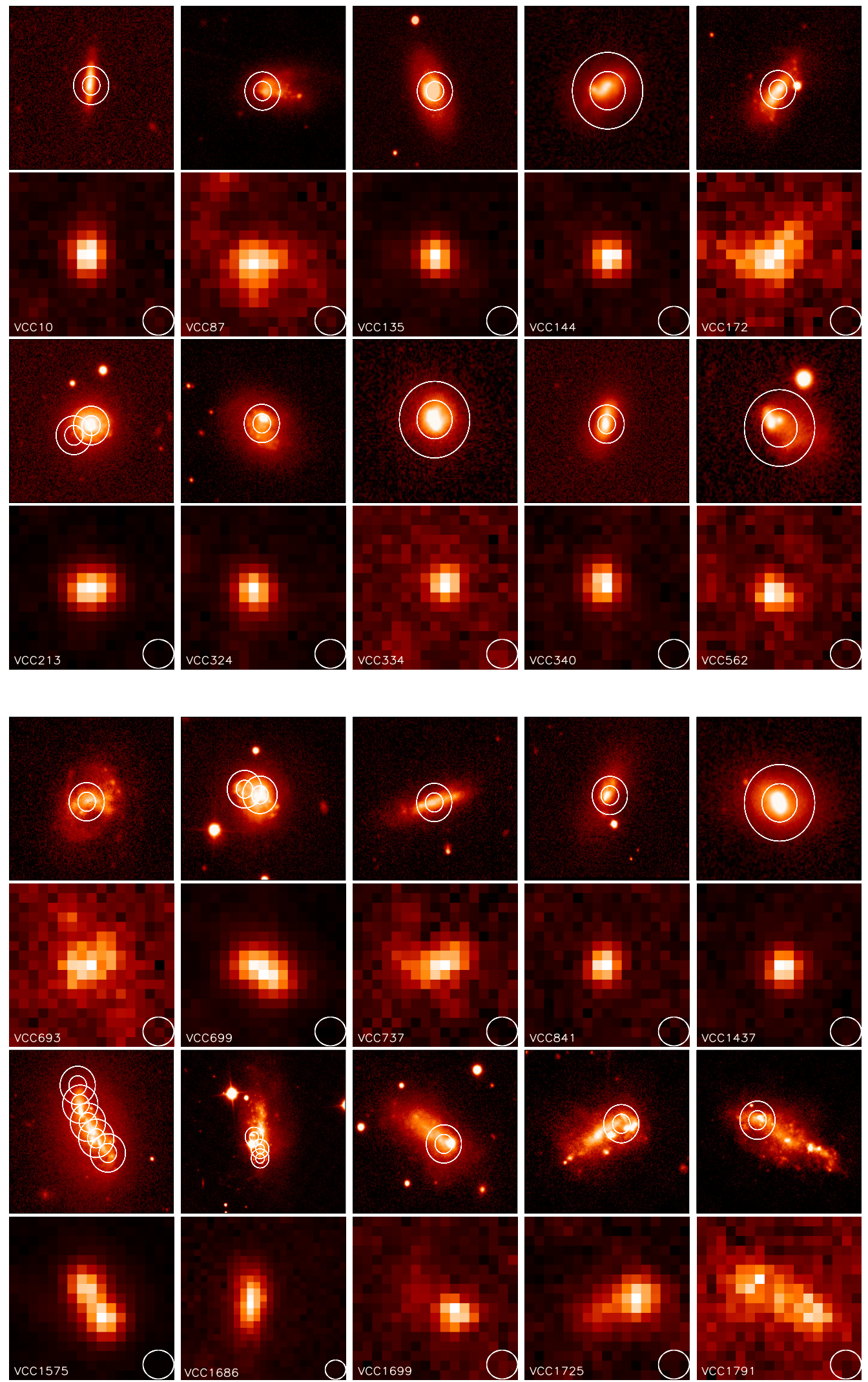

Fig. 2. Sample of Virgo SFDs observed at IRAM: the 30-m telescope pointings are overlaid on the SDSS $g$ images (first and third rows). The larger and smaller circles indicate the beam sizes at $115 \mathrm{GHz}$ and $230 \mathrm{GHz}$, respectively. Herschel/SPIRE $250 \mu \mathrm{m}$ images are also displayed for each target (second and fourth rows) with the $250 \mu \mathrm{m}$ beam size shown in the bottom right corner. 
Table 2. CO line intensities, systemic velocities, line widths, and noise level at 115 and $230 \mathrm{GHz}$.

\begin{tabular}{|c|c|c|c|c|c|c|c|c|c|}
\hline ID & $\begin{array}{c}I_{\mathrm{CO}, w}^{(1-0) a} \\
{\left[\mathrm{~K} \mathrm{~km} \mathrm{~s}^{-1}\right]}\end{array}$ & $\begin{array}{c}I_{\mathrm{CO}, g}^{(1-0) b} \\
{\left[\mathrm{~K} \mathrm{~km} \mathrm{~s}^{-1}\right]}\end{array}$ & $\begin{array}{c}I_{\mathrm{CO}, w}^{(2-1) a} \\
{\left[\mathrm{~K} \mathrm{~km} \mathrm{~s}^{-1}\right]}\end{array}$ & $\begin{array}{c}I_{\mathrm{CO}, g}^{(2-1) b} \\
{\left[\mathrm{~K} \mathrm{~km} \mathrm{~s}^{-1}\right]}\end{array}$ & $\begin{array}{c}V_{\text {sys }, g}{ }^{c} \\
{\left[\mathrm{~km} \mathrm{~s}^{-1}\right]}\end{array}$ & $\begin{array}{c}\Delta V_{g}^{(1-0)} \\
{\left[\mathrm{km} \mathrm{s}^{-1}\right]}\end{array}$ & $\begin{array}{c}\Delta V_{g}^{(2-1)} \\
{\left[\mathrm{km} \mathrm{s}^{-1}\right]}\end{array}$ & $\begin{array}{c}\sigma^{(1-0) d} \\
{[\mathrm{mK}]}\end{array}$ & $\begin{array}{c}\sigma^{(2-1) d} \\
{[\mathrm{mK}]}\end{array}$ \\
\hline VCC10 & $<0.41$ & $<0.41$ & $0.99 \pm 0.13$ & $1.11 \pm 0.19$ & $-; 1938$ & - & 59 & 4.7 & 5.0 \\
\hline VCC87 & $<0.42$ & $<0.42$ & - & - & -157 & - & - & 3.7 & - \\
\hline VCC135 & $1.71 \pm 0.30$ & $1.84 \pm 0.34$ & $5.25 \pm 0.40$ & $5.37 \pm 0.47$ & $2409 ; 2406$ & 112 & 112 & 9.4 & 10.8 \\
\hline VCC144 & $<0.39$ & $<0.39$ & $1.11 \pm 0.12$ & $1.13 \pm 0.14$ & $-; 2026$ & - & 69 & 4.1 & 4.0 \\
\hline VCC172 & $<1.06$ & $<1.06$ & $<1.26$ & $<1.26$ & 2175 & - & - & 8.3 & 9.9 \\
\hline VCC213(a) & $3.57 \pm 0.25$ & $3.44 \pm 0.26$ & $3.08 \pm 0.33$ & $3.66 \pm 0.31$ & $-164 ;-167$ & 80 & 69 & 10.3 & 11.3 \\
\hline VCC213(b) & $2.45 \pm 0.19$ & $2.64 \pm 0.23$ & $3.64 \pm 0.21$ & $3.54 \pm 0.25$ & $-131 ;-125$ & 44 & 29 & 6.0 & 9.4 \\
\hline VCC 324 & $0.51 \pm 0.11$ & $0.47 \pm 0.16$ & $0.74 \pm 0.09$ & $0.69 \pm 0.08$ & $1540 ; 1530$ & 42 & 26 & $2.8^{e}$ & 3.3 \\
\hline VCC334 & $<0.22$ & $<0.22$ & - & - & -240 & - & - & 3.0 & - \\
\hline VCC340 & $0.58 \pm 0.13$ & $0.62 \pm 0.16$ & $0.90 \pm 0.11$ & $0.81 \pm 0.08$ & $1519 ; 1513$ & 46 & 32 & 4.0 & 3.5 \\
\hline VCC562 & $<0.80$ & $<0.80$ & - & - & 44 & - & - & 10.9 & - \\
\hline VCC693 & $<0.58$ & $<0.58$ & $<0.55$ & $<0.55$ & 2048 & - & - & 5.1 & 4.9 \\
\hline VCC699(a) & $0.66 \pm 0.13$ & $0.80 \pm 0.13$ & $0.65 \pm 0.09$ & $0.63 \pm 0.09$ & $720 ; 711$ & 69 & 29 & 4.4 & 4.0 \\
\hline VCC699(b) & $0.65 \pm 0.13$ & $0.64 \pm 0.23$ & $0.60 \pm 0.11$ & $0.55 \pm 0.15$ & $743 ; 734$ & 48 & 17 & 6.2 & 7.5 \\
\hline VCC737 & $<0.39$ & $<0.39$ & - & - & 1725 & - & - & 2.7 & - \\
\hline VCC 841 & $<0.31$ & $<0.31$ & - & - & 491 & - & - & 4.4 & - \\
\hline VCC1437 & $1.25 \pm 0.13$ & $1.29 \pm 0.17$ & $2.62 \pm 0.12$ & $2.74 \pm 0.11$ & $1157 ; 1160$ & 56 & 51 & 4.8 & 3.8 \\
\hline VCC1575(a) & $3.21 \pm 0.22$ & $3.33 \pm 0.23$ & $5.54 \pm 0.26$ & $5.26 \pm 0.27$ & $577 ; 579$ & 39 & 32 & 7.7 & 10.6 \\
\hline VCC1575(b) & $4.48 \pm 0.23$ & $4.51 \pm 0.27$ & $6.95 \pm 0.30$ & $6.83 \pm 0.34$ & $583 ; 583$ & 48 & 43 & 8.5 & 11.4 \\
\hline VCC $1575(c)$ & $4.83 \pm 0.25$ & $5.11 \pm 0.25$ & $3.93 \pm 0.21$ & $3.80 \pm 0.22$ & $608 ; 610$ & 50 & 32 & 6.4 & 9.5 \\
\hline VCC1575(d) & $4.41 \pm 0.19$ & $4.38 \pm 0.22$ & $6.38 \pm 0.23$ & $6.66 \pm 0.24$ & $621 ; 616$ & 42 & 42 & 7.6 & 9.6 \\
\hline VCC $1575(\mathrm{e})$ & $1.20 \pm 0.20$ & $0.91 \pm 0.16$ & $<0.50$ & $<0.50$ & $574 ;-$ & 20 & - & 7.6 & 9.7 \\
\hline VCC1686(a) & $0.74 \pm 0.12$ & $0.90 \pm 0.13$ & $0.81 \pm 0.11$ & $0.83 \pm 0.14$ & $1123 ; 1121$ & 37 & 28 & 5.8 & 6.4 \\
\hline VCC1686(b) & $<0.90$ & $<0.90$ & $<0.96$ & $<0.96$ & $-;-$ & - & - & 9.5 & 10.0 \\
\hline VCC1686(c) & $1.20 \pm 0.30$ & $1.31 \pm 0.41$ & $<1.06$ & $<1.06$ & 1130;- & 70 & - & $6.3^{e}$ & 11.1 \\
\hline VCC1699 & $<0.30$ & $<0.30$ & - & - & 1632 & - & - & 2.8 & - \\
\hline VCC1725 & $0.41 \pm 0.12$ & $0.46 \pm 0.13$ & $<0.34$ & $<0.34$ & $1049 ;-$ & 28 & - & 4.5 & 5.6 \\
\hline VCC1791 & $<0.46$ & $<0.46$ & - & - & 2075 & - & - & 4.2 & - \\
\hline
\end{tabular}

Notes. ${ }^{(a)}$ Measured summing of the spectrum over the observed velocity width. ${ }^{(b)}$ Measured by fitting a Gaussian function or a linear combination of the first two symmetric Hermite functions. ${ }^{(c)}$ Systemic velocity derived from the fit of the $\mathrm{CO}(1-0)$ and $\mathrm{CO}(2-0)$ lines. For non-detections we display only the systemic velocities determined from the SDSS optical spectra. ${ }^{(d)}$ Noise level at a spectral resolution of $8.1 \mathrm{~km} \mathrm{~s}^{-1}$. ${ }^{(e)}$ Noise level measured at a spectral resolution of $16.2 \mathrm{~km} \mathrm{~s}^{-1}$.

where $D_{25}$ is the diameter at the 25 th $B$-magnitude isophote in arcseconds, $S_{\mathrm{CO}}^{\mathrm{central}}$ is the measured flux that is related to the integrated line intensity by $S_{\mathrm{CO}}^{\text {central }}=G \times I_{\mathrm{CO}}^{\mathrm{central}}$, and $G$ is the gain of the telescope.

The second method, introduced by Lisenfeld et al. (2011), provides a more analytic approach to derive the aperture correction. The starting assumption is that the radial surface distribution of the $\mathrm{CO}$ emission $S_{\mathrm{CO}}(r)$ follows an exponential law with a scale length $r_{\mathrm{e}}$, which is related to the radius at the 25th $B$-magnitude isophote, $r_{25}$, according to $r_{\mathrm{e}}=0.2 r_{25}$ (Leroy et al. 2008; Lisenfeld et al. 2011). The total CO flux, $S_{\text {CO }}^{\text {tot }}$, is calculated by spatially integrating the $\mathrm{CO}$ exponential profile (see Eq. (4) in Lisenfeld et al. 2011). $S_{\text {CO }}^{\text {central }}$ is derived by convolving the $\mathrm{CO}$ exponential distribution with a Gaussian beam (see Eq. (5) in Lisenfeld et al. 2011). The aperture correction, $f_{\text {ap }}$, in this case depends on the ratio of the scale length and the beam size, $r_{\mathrm{e}} / \theta_{\text {beam }}$, as well as on the galaxy inclination $i$ (Lisenfeld et al. 2011).

We calculated the aperture corrections for both methods and obtained comparable values ranging between 1.2 and 4.4. The largest discrepancies are found in the most extended objects of the sample (VCC1575, VCC1686, VCC1699, VCC1725: see Table A.2). Comparing the extrapolation of a single-beam observations to complete CO maps, Boselli et al. (2014c) concluded that the analytic prescription of Lisenfeld et al. is more appropriate than the empirical relation of Saintonge et al. (2011). Moreover, the COLD GASS sample consisted of massive $\left(>10^{10} M_{\odot}\right)$ and metal-rich galaxies, fairly different from our sample of lowmass dwarfs with intermediate metallicities. Therefore in the rest of this work we adopt the method of Lisenfeld et al. (2011) to correct for extended emission, although this choice does not significantly affect the estimate of the final $\mathrm{H}_{2}$ masses ${ }^{7}$.

\subsubsection{Multiple-pointing observations}

For galaxies with more than one pointed observation, hereafter extended sources, we computed the total $\mathrm{CO}$ flux by fitting the radial surface distribution of the emission. We assumed that the emitting region fills the beam and that the brightness decreases exponentially with radius (Leroy et al. 2009b). At each observed position we convolved the modelled brightness distribution with

\footnotetext{
7 We note that the single $\mathrm{CO}$ pointing is offset from the centre of the galaxy in three objects where we chose to target the peaks of the FIR emission. Two galaxies were not detected (VCC1791 and VCC1699), and one (VCC1725) is a tentative detection. Applying these techniques, which are calibrated for a central pointing, means that we are obtaining more conservative upper limits for VCC1791 and VCC1699, and that we are slightly overestimating the total CO intensity of VCC1725.
} 
the telescope beam and integrated over the beam extent to derive the predicted line intensity. We compared the modelled to the observed flux and determined the exponential scale length that best fitted the data using a $\chi^{2}$ test. We followed this procedure for both the $J=1-0$ and $J=2-1$ lines. A flat brightness distribution provided the best fit to the $J=2-1$ emission of VCC213 and to the $J=1-0$ emission of VCC699. In these cases we only integrated out to 33 arcsec (equivalent to 1.5 times the FWHM of the $115 \mathrm{GHz}$ beam) to determine the total $\mathrm{CO}$ flux. We compared these results to the method described in the previous subsection and obtained similar results within the uncertainties.

\subsection{Correction for a source size smaller than the beam}

The underlying assumption in the calculation of the aperture corrections derived in Sect. 3.1 is that the emission fills the beam. If the angular size of a source, $\Omega_{\mathrm{s}}$, is smaller than the beam size, $\Omega_{\text {beam }}$, the main beam temperature, $T_{\mathrm{mb}}$, will be lower than the line brightness temperature, $T_{\mathrm{B}}$, by a factor $\Omega_{\mathrm{s}} / \Omega_{\text {beam }}$. In this case the observed integrated line intensity, $I_{\mathrm{CO}}=\int T_{\mathrm{mb}} \mathrm{d} v$, measures the beam diluted brightness temperature, while the intrinsic $\mathrm{CO}$ line intensity is the source brightness temperature integrated over the velocity width. Here we describe a method to estimate the size of the emitting region based on the comparison between the observed main beam temperature line ratio, $I_{2-1} / I_{1-0}$, and the intrinsic brightness temperature ratio $R_{21} \equiv T_{\mathrm{B}}^{2-1} / T_{\mathrm{B}}^{1-0}$. We assume that the source geometry is described by either a uniform disc or a Gaussian, and we determine the corresponding corrections factors.

\subsubsection{Estimate of source sizes}

For a uniformly bright disc of radius $R_{\mathrm{u}}$ and angular size $\Omega_{\mathrm{u}}=$ $\pi R_{\mathrm{u}}^{2}$, the main beam temperature to brightness temperature ratio is given by (Heeschen 1961; Corbelli et al. 2011)

$\frac{T_{\mathrm{mb}}}{T_{\mathrm{B}}}=1-\mathrm{e}^{-\left(R_{\mathrm{u}} / 0.6 \theta_{\text {beam }}\right)^{2}}=1-\mathrm{e}^{-\Omega_{\mathrm{u}} / \Omega_{\text {beam }}}$,

where $\Omega_{\text {beam }}=1.13 \theta_{\text {beam }}^{2}$ is the effective solid angle for a Gaussian beam of FWHM $\theta_{\text {beam }}$. For a Gaussian source of angular size $\Omega_{\mathrm{g}}=1.13 \theta_{\mathrm{g}}^{2}$ (and FWHM $\theta_{\mathrm{g}}$ ), this ratio can be written as (Bell 1993)

$$
\frac{T_{\mathrm{mb}}}{T_{\mathrm{B}}}=\frac{\Omega_{\mathrm{g}}}{\Omega_{\mathrm{g}}+\Omega_{\text {beam }}} .
$$

We can then use the observed main beam temperature line ratio, $I_{2-1} / I_{1-0}$, to estimate the size of the emitting region. For a uniform and a Gaussian brightness distribution we have

$$
\begin{aligned}
& \frac{I_{2-1}}{I_{1-0}}=R_{21} \frac{1-\mathrm{e}^{-\Omega_{\mathrm{u}} / \Omega_{2-1}}}{1-\mathrm{e}^{-\Omega_{\mathrm{u}} / \Omega_{1-0}}} \\
& \frac{I_{2-1}}{I_{1-0}}=R_{21} \frac{\Omega_{\mathrm{g}}+\Omega_{1-0}}{\Omega_{\mathrm{g}}+\Omega_{2-1}},
\end{aligned}
$$

where we assumed $R_{21}=0.8$, the intrinsic line ratio typically observed in nearby resolved galaxies (Leroy et al. 2009b). Because $\Omega_{\text {beam }}^{1-0} / \Omega_{\text {beam }}^{2-1}=4$ for the IRAM 30-m telescope, $\frac{I_{2-1}}{I_{1-0}}$ can be up to a factor 4 higher than the brightness temperature line ratio (if $\left.\Omega_{\mathrm{u}}, \Omega_{\mathrm{g}} \ll \Omega_{\text {beam }}\right)$, while for an emitting region larger than the beam the two ratios will be equal $\left(\frac{I_{2-1}}{I_{1-0}} \simeq R_{21}\right)$

When both lines were detected, we derived a range of size estimates from Eqs. (8) and (9), considering that the observed integrated intensities of the two lines vary between $I_{\mathrm{CO}}-\delta I_{\mathrm{CO}}$ and $I_{\mathrm{CO}}+\delta I_{\mathrm{CO}}$, where $\delta I_{\mathrm{CO}}$ is the measured uncertainty. Then we took the mean value of the upper and lower estimates of both cases. When only $\mathrm{CO}(2-1)$ was detected (as in VCC10 and VCC144), we only set upper limits to the source size. In this case we considered the whole range of sizes compatible with the observations (from point-like to the maximum value defined by the upper limit) and took the mean of the derived estimates. The estimated sizes for a uniform and a Gaussian brightness distribution $\left(2 R_{\mathrm{u}}, \theta_{\mathrm{g}}\right)$ are displayed in Table A.2.

\subsubsection{Correction factors}

When the sizes were known, we determined the corresponding correction factors to the flux, $f_{\mathrm{s}}^{\mathrm{u}, \mathrm{g}}$, which account for the non-negligible extension of the source compared to the beam FWHM (Heeschen 1961). For a uniformly bright disc $\left(\Omega_{\mathrm{u}}\right)$ and a Gaussian source $\left(\Omega_{\mathrm{g}}\right)$, we define using Eqs. (6) and (7)

$$
\begin{aligned}
& f_{\mathrm{s}}^{\mathrm{u}}=\frac{\left(\frac{\Omega_{\mathrm{u}}}{\Omega_{\text {beam }}}\right)}{\left(1-\mathrm{e}^{-\left(\Omega_{\mathrm{u}} / \Omega_{\text {beam }}\right)}\right)} \\
& f_{\mathrm{s}}^{\mathrm{g}}=\left(1+\frac{\Omega_{\mathrm{g}}}{\Omega_{\text {beam }}}\right),
\end{aligned}
$$

where $\Omega_{\text {beam }}$ corresponds to the IRAM 30-m telescope beam at $115 \mathrm{GHz}$. For point-like sources $\left(\Omega_{\mathrm{u}}, \Omega_{\mathrm{g}} \ll \Omega_{\text {beam }}\right)$ the correction is negligible $\left(f_{\mathrm{s}}^{\mathrm{u}}=f_{\mathrm{s}}^{\mathrm{g}} \rightarrow 1\right)$, while the highest values are obtained for sizes comparable to the beam. The correction factors $f_{\mathrm{s}}^{\mathrm{u}}, f_{\mathrm{s}}^{\mathrm{g}}$ are given in Table A.2.

\subsection{Final CO fluxes}

After assessing the different correction factors that take into account the finite source size $\left(S_{\mathrm{s}}^{\text {tot }}=f_{\mathrm{s}}^{\mathrm{u}, \mathrm{g}} G I_{\mathrm{CO}}^{\text {beam }}\right)$ and that the emitting region can be more extended than the beam $\left(S_{\text {Ext }}^{\text {tot }}=\right.$ $\left.f_{\text {ap }} G I_{\mathrm{CO}}^{\text {beam }}\right)$, the total $\mathrm{CO}$ fluxes were computed as the average between the minimum and maximum flux obtained for all possible sizes. The difference between the mean and extreme values defines the one-sigma uncertainty. For VCC135 we considered only the correction for source size smaller than the beam because of its small extent. When no lines were detected, we converted the upper limit to the main beam temperature, estimated with Eq. (2), to a flux upper limit by multiplying it by the gain and by the aperture correction for extended regions derived in Sect. 3.1 (see Table A.2).

\section{CO detections versus non-detections}

Figure 1 compares the global properties of galaxies with a detected $\mathrm{CO}$ emission with those of non-detections. The main parameter that appears to separate detections from non-detections is stellar mass: more massive dwarf galaxies are more likely to be detected in CO (Albrecht et al. 2004; Israel 2005; Leroy et al. 2005). The detected galaxies have stellar masses in between the Large and Small Magellanic Cloud, with $\log \left(M_{*} / M_{\odot}\right)=$ 9.3 (van der Marel et al. 2002) and 8.5 (Harris \& Zaritsky 2004), respectively. Our dwarfs lie along the main-sequence relation of star-forming galaxies (Brinchmann et al. 2004; Chang et al. 2015), therefore, the detections also have overall the highest SFRs. The majority of the detections also correspond to galaxies with the lowest HI fractions $\left(M_{\mathrm{HI}} / M_{*}\right)$, which is usually found 

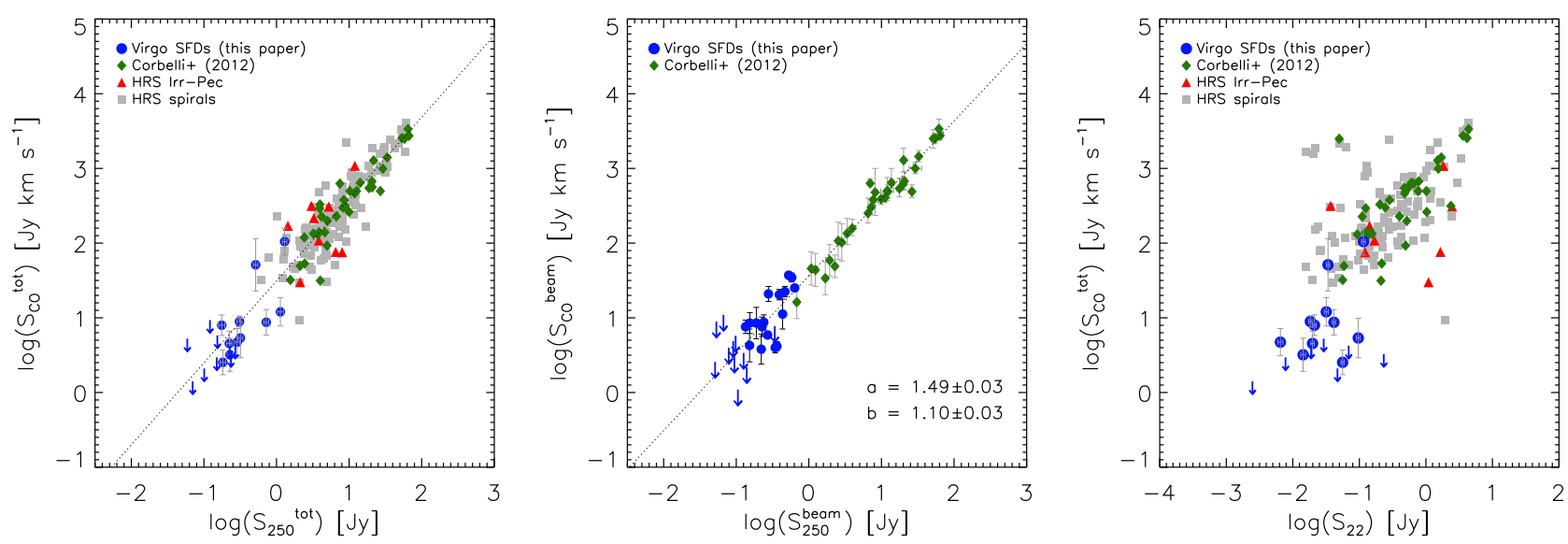

Fig. 3. Left: total $\mathrm{CO}(1-0)$ fluxes against total Herschel/SPIRE flux densities at $250 \mu \mathrm{m}$. Virgo SFD dwarfs (blue dots) are compared to Virgo spirals (green diamonds) from Corbelli et al. (2012), and spiral and irregular or peculiar galaxies from the HRS survey (grey squares and red triangles, respectively). The dotted line shows the best fit to the three samples. Centre: $\mathrm{CO}(1-0)$ fluxes within the $\sim 22^{\prime \prime}$ IRAM 30-m telescope beam against $250 \mu \mathrm{m}$ flux densities measured within the same area. Here only Virgo SFD and spiral galaxies are shown. Right: total CO(1-0) fluxes against total WISE flux densities at $22 \mu \mathrm{m}$ for Virgo and HRS samples. Symbols are the same as in the left panel.

to be anti-correlated with stellar mass (Lara-López et al. 2010; Huang et al. 2012; Gavazzi et al. 2013). The higher CO detection rate at higher $M_{*}$ can be easily understood. With increasing stellar mass, all global quantities related to the inner disc increase, including the $\mathrm{CO}$ line intensity; moreover, a higher stellar density favours the formation of molecules (Elmegreen 1993). This is a consequence of the higher potential well in the inner disc that is provided by the stellar component, which favours higher gas densities and higher $\mathrm{H}_{2}$ formation rates, as we discuss in Sect. 7.2. Non-detections are more HI-rich than detections. Lower stellar surface densities lead to a more diffuse, lowpressure distribution of atomic gas, which is a less suitable environment for converting atomic into molecular gas (Leroy et al. 2005).

The detection rate increases in the higher metallicity bins, but we caution about the uncertainties in deriving the metal abundances from the SDSS spectra that lack the [O II] $\lambda 3727$ line (see Grossi et al. 2015 for details). The galaxy with the lowest metallicity detected in our survey is $\operatorname{VCC} 324$ with $12+\log (\mathrm{O} / \mathrm{H})=$ $8.14 \pm 0.1$.

Grossi et al. (2015) discussed that the whole sample of FIRdetected SFDs is preferentially located in the less dense regions of the cluster. In the sub-sample that we observed at IRAM, only one dwarf, VCC1686, is within two degrees of M87. The others are distributed in the outskirts of subcluster B (centred on M49), in the low-velocity cloud (LVC), a subgroup of galaxies at $V \lesssim 0 \mathrm{~km} \mathrm{~s}^{-1}$ superposed on the M87 region that is thought to be infalling towards the cluster core from behind (Hoffman et al. 1989), and in the Virgo southern extension. $\mathrm{Six}^{8}$ out of 20 galaxies are at larger distances, and they are located in the background structures called $\mathrm{W}^{\prime}, \mathrm{W}$, and $\mathrm{M}$ clouds to the south-west and to the north-west of the cluster core, respectively (Ftaclas et al. 1984; Binggeli et al. 1987). These structures are outside the Virgo virial radius and represent an intermediate density environment. It is not possible to relate the lack of $\mathrm{CO}$ emission in the non-detected galaxies to their location within the cluster, since they show a projected spatial distribution similar to the CO-detected dwarfs.

\footnotetext{
8 VCC10, VCC135, VCC144, VCC172, VCC340, VCC699.
}

\section{FIR-CO correlation}

The combined analysis of $\mathrm{HeViCS}$ data and $\mathrm{CO}(1-0)$ data for a sample of spiral galaxies in Virgo has shown that a linear relation holds between cold dust emission and $\mathrm{CO}$ brightness down to $F_{250}=2.5 \mathrm{Jy}$ and $S_{\mathrm{CO}}=100 \mathrm{Jy} \mathrm{km} \mathrm{s}{ }^{-1}$ (Corbelli et al. 2012). The main sample examined in this work comprised spiral galaxies with metallicities above solar. Here we wish to assess whether the correlation holds at lower stellar masses and subsolar metallicities.

In Fig. 3 (left panel) we plot the observed total CO fluxes $\left(S_{\mathrm{CO}}^{\text {tot }}\right)$ against Herschel/SPIRE flux densities at $250 \mu \mathrm{m}\left(S_{250}^{\text {tot }}\right)$ for our sample. For those galaxies with only a $\mathrm{CO}(2-1)$ detection, we estimated the $\mathrm{CO}(1-0)$ flux assuming a $(2-1) /(1-0)$ ratio of 0.8 (Leroy et al. 2009b), after taking into account corrections for an emitting region smaller or larger than the beam (see Sect. 3). Flux densities at $250 \mu \mathrm{m}$ were taken from Grossi et al. (2015). In the figure we compare our galaxies to two samples from the literature: i) the Virgo spirals of Corbelli et al. (2012) and ii) the Herschel Reference Survey (HRS; Boselli et al. 2010), a volume-limited (15 Mpc $\leq D \leq 25 \mathrm{Mpc}$ ), $K$-band-selected set of 323 galaxies, of which 260 with morphology later than Sa and 63 with morphological types S0 and earlier. In this and the following figures we use only the HRS late-type galaxies as a comparison sample, which we divided for simplicity into two morphological bins: spirals (from Sa to $\mathrm{Sd}$ ) and irregulars-peculiars (later than $\mathrm{Sd}$ ), which are expected to have more similar properties to the Virgo SFDs. HRS CO fluxes were measured in Boselli et al. (2014a) and $250 \mu \mathrm{m}$ flux densities in Ciesla et al. (2012) ${ }^{9}$. The same quantities for Virgo spirals were taken from Corbelli et al. (2012) ${ }^{9}$.

CO fluxes correlate strongly with FIR emission even at the lower stellar masses probed by our sample of dwarfs. The correlation extends over three orders of magnitude in both $S_{\mathrm{CO}}^{\text {tot }}$ and $S_{250}^{\text {tot }}$ (at least for galaxies with subsolar metallicities), and it is close to linear, as found by Corbelli et al. (2012). The best-fit relation for the three data sets is

$\log S_{\mathrm{CO}}^{\text {tot }}=1.49( \pm 0.03)+1.10( \pm 0.03) \log S_{250}^{\text {tot }}$,

9 FIR flux densities were corrected for the revised SPIRE beam areas and calibration similarly to what was done with the HeViCS data in Grossi et al. (2015). 
and it is plotted in the left panel of Fig. $3^{10}$. In the middle panel of the figure we show the $\mathrm{CO}(1-0)$ flux in single-pointing observations (corrected for a finite source size) and $S_{250}^{\text {beam }}$ measured in circular apertures of $18^{\prime \prime} 6$ radius $^{11}$, centred at the same positions as the IRAM pointings (see Table A.1). The best-fit relation in this case has the same intercept and slope as the one determined for the total FIR and CO fluxes (central panel of Fig. 3). The weighted mean of the scatter of the Virgo SFD data about the best fit (see Eq. (6) in Williams et al. 2010) is about three times lower when the single pointings are considered because in this case the uncertainties related to the global content of $\mathrm{CO}$ are not taken into account.

Schruba et al. (2011) showed that spiral galaxies from the HERACLES survey (Leroy et al. 2009b) exhibit a strong correlation between $\mathrm{CO}$ and $24 \mu \mathrm{m}$ emission because of the tight link between molecular gas and star formation that is traced by mid-infrared (MIR) radiation at $24 \mu \mathrm{m}$. As a comparison, in the right panel of Fig. 3 we display $S_{\mathrm{CO}}$ against the flux density at $22 \mu \mathrm{m}, S_{22}$, obtained from the Wide-field Infrared Survey Explorer (WISE; Wright et al. 2010) for all samples. We used $22 \mu \mathrm{m}$ emission instead of $24 \mu \mathrm{m}$ because WISE fluxes are available for the three sets of galaxies. WISE flux densities were taken from Boselli et al. (2014d) for the HRS and Virgo spiral galaxies and from Grossi et al. (2015) for the Virgo SFDs. The figure shows a trend between $S_{\text {CO }}^{\text {tot }}$ and $S_{22}$, but the scatter is larger than for the $S_{\mathrm{CO}}^{\text {tot }}-S_{250}$ correlation both at high and low stellar masses. Therefore $\mathrm{CO}$ emission is consistent with being linearly proportional to FIR emission down to the low stellar masses probed by our sample of dwarf galaxies; the link between molecular gas and cold dust appears to be stronger than that with warm dust traced by MIR emission.

\section{Estimates of molecular hydrogen masses}

The conversion between $\mathrm{CO}$ intensities and $\mathrm{H}_{2}$ column density is usually performed using a CO-to- $\mathrm{H}_{2}$ conversion factor, $X_{\mathrm{CO}}$, which is defined as

$X_{\mathrm{CO}}=\frac{N\left(\mathrm{H}_{2}\right)}{I_{\mathrm{CO}}}\left[\mathrm{cm}^{-2}\left(\mathrm{~K} \mathrm{~km} \mathrm{~s}^{-1}\right)^{-1}\right]$.

In the Milky Way the standard conversion factor is $X_{\mathrm{CO}}^{\mathrm{gal}}=2 \times$ $10^{20} \mathrm{~cm}^{-2}\left(\mathrm{~K} \mathrm{~km} \mathrm{~s}^{-1}\right.$ (Dame et al. 2001; Lombardi et al. 2006; Bolatto et al. 2013, and references therein), equivalent to $\alpha_{\mathrm{CO}}^{\mathrm{gal}}=$ $3.21 M_{\odot} \mathrm{pc}^{-2}\left(\mathrm{~K} \mathrm{~km} \mathrm{~s}^{-1}\right)^{-1}$ without the correction for helium.

$X_{\mathrm{CO}}$ is expected to vary with the physical properties of the ISM, such as metal content, ionising radiation field strength, and gas surface density. Metallicity is the dominant parameter affecting $X_{\mathrm{CO}}$ variations, however, since it is found to increase sharply in systems with metallicities below $12+\log [\mathrm{O} / \mathrm{H}] \approx 8.4$, or onehalf the solar value (Israel 1997; Taylor et al. 1998; Mizuno et al. 2001; Boselli et al. 2002; Schruba et al. 2012). Thus, applying a galactic CO-to- $\mathrm{H}_{2}$ conversion factor can lead to underestimating the molecular gas content in low-metallicity galaxies.

For this reason, we compare two different methods to estimate $\mathrm{H}_{2}$ masses that take into account the variation of the $X_{\mathrm{CO}}$ factor with the metal content.

1. We use a metallicity-dependent CO-to- $\mathrm{H}_{2}$ conversion factor based on the model of Wolfire et al. (2010), $\alpha_{\mathrm{CO}}^{\mathrm{W} 10}$, which

${ }^{10}$ We also fitted the data on linear scales and found a best-fit line log $S_{\mathrm{CO}}^{\text {tot }}=-37.4( \pm 14.6)+44.4( \pm 1.8) \log \left(S_{250}^{\text {tot }}\right)$.

${ }_{11}$ The circular aperture chosen for the $250 \mu \mathrm{m}$ photometry corresponds to $88 \%$ of the effective area of the $30-\mathrm{m}$ telescope beam at $115 \mathrm{GHz}$.

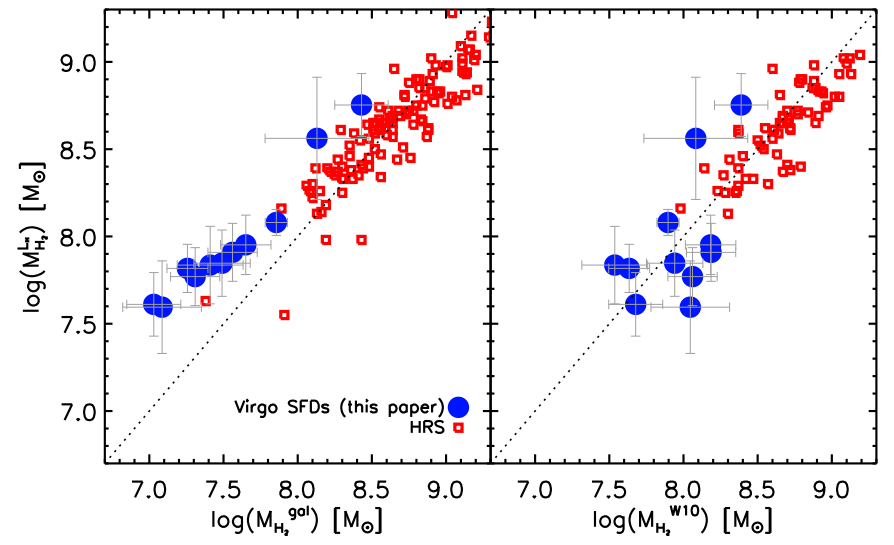

Fig. 4. Comparison between $\mathrm{H}_{2}$ masses derived with different methods. The $H$-luminosity-dependent estimate (Boselli et al. 2002) is plotted against the values obtained with a Milky Way standard conversion factor (left) and a metallicity-dependent $X$ factor (Wolfire et al. 2010, right panel). As a comparison we also show galaxies from the HRS survey (red squares). The dotted lines indicate the one-to-one relation.

includes the possiblilty that a fraction of $\mathrm{H}_{2}$ mass may lie outside the $\mathrm{CO}$ region, where carbon is in the atomic or ionised state:

$\frac{\alpha_{\mathrm{CO}}^{\mathrm{W} 10}}{\alpha_{\mathrm{CO}}^{\mathrm{gal}}}=\exp \left(\frac{0.74-0.078 \ln Z^{\prime}}{Z^{\prime}}\right) \times \exp \left(-0.74+0.078 \ln Z^{\prime}\right)$,

where $Z^{\prime}=Z / Z_{\odot}=10^{(12+\log (\mathrm{O} / \mathrm{H})-8.7)}$, and $\alpha_{\mathrm{CO}}^{\mathrm{MW}}$ is the Milky Way conversion factor in units of $M_{\odot} \mathrm{pc}^{-2}\left(\mathrm{~K} \mathrm{~km} \mathrm{~s}^{-1}\right)^{-1}$.

2. We also consider luminosity-dependent $X_{\mathrm{CO}}$ (Boselli et al. 2002), based on the luminosity-metallicity relation

$\log \frac{X_{\mathrm{CO}}^{L_{H}}}{\mathrm{~cm}^{-2}\left(\mathrm{~K} \mathrm{~km} \mathrm{~s}^{-1}\right)^{-1}}=-0.38 \log L_{H}+24.23$,

where $L_{H}$ is the total galaxy luminosity in the $H$ band. This method was applied by Boselli et al. (2014a) to estimate molecular gas masses in the HRS galaxies, which we use in the following sections as a comparison sample to the $\mathrm{H}_{2}$ properties of the Virgo SFDs.

We calculated the molecular hydrogen mass of the Virgo SFD galaxies from the following relation (Solomon \& Vanden Bout 2005):

$M_{\mathrm{H}_{2}}=3.25 \times 10^{7} \alpha_{\mathrm{CO}}^{i} S_{\mathrm{CO}} v^{-2} D^{2}\left[M_{\odot}\right]$,

where $\alpha_{\mathrm{CO}}^{i}=\left[\alpha_{\mathrm{CO}}^{\mathrm{gal}}, \alpha_{\mathrm{CO}}^{L_{H}}, \alpha_{\mathrm{CO}}^{\mathrm{W} 10}\right], S_{\mathrm{CO}}$ is the $\mathrm{CO}(1-0)$ flux in Jy $\mathrm{km} \mathrm{s}^{-1}$ corrected for the extension of the emitting region (smaller or larger than the beam; see Sect. 3.3), $v$ the frequency of the $\mathrm{CO}(1-0)$ line in $\mathrm{GHz}$, and $D$ the distance to the galaxy in Mpc.

In Fig. 4 we compare the $\mathrm{H}_{2}$ masses derived with a $H$-luminosity-dependent $X_{\mathrm{CO}}$ to those obtained with a galactic (left panel) and a metallicity-dependent (right panel), $X_{\mathrm{CO}}$. As expected, when the $H$-luminosity (or metallicity dependence) is taken into account, we obtain higher molecular masses than with the assumption of a fixed $\mathrm{X}_{\mathrm{CO}}$. The $X$ factor of Boselli et al. (2002) is at most four times larger than the galactic value, while the calibration of Wolfire et al. (2010) gives masses higher 

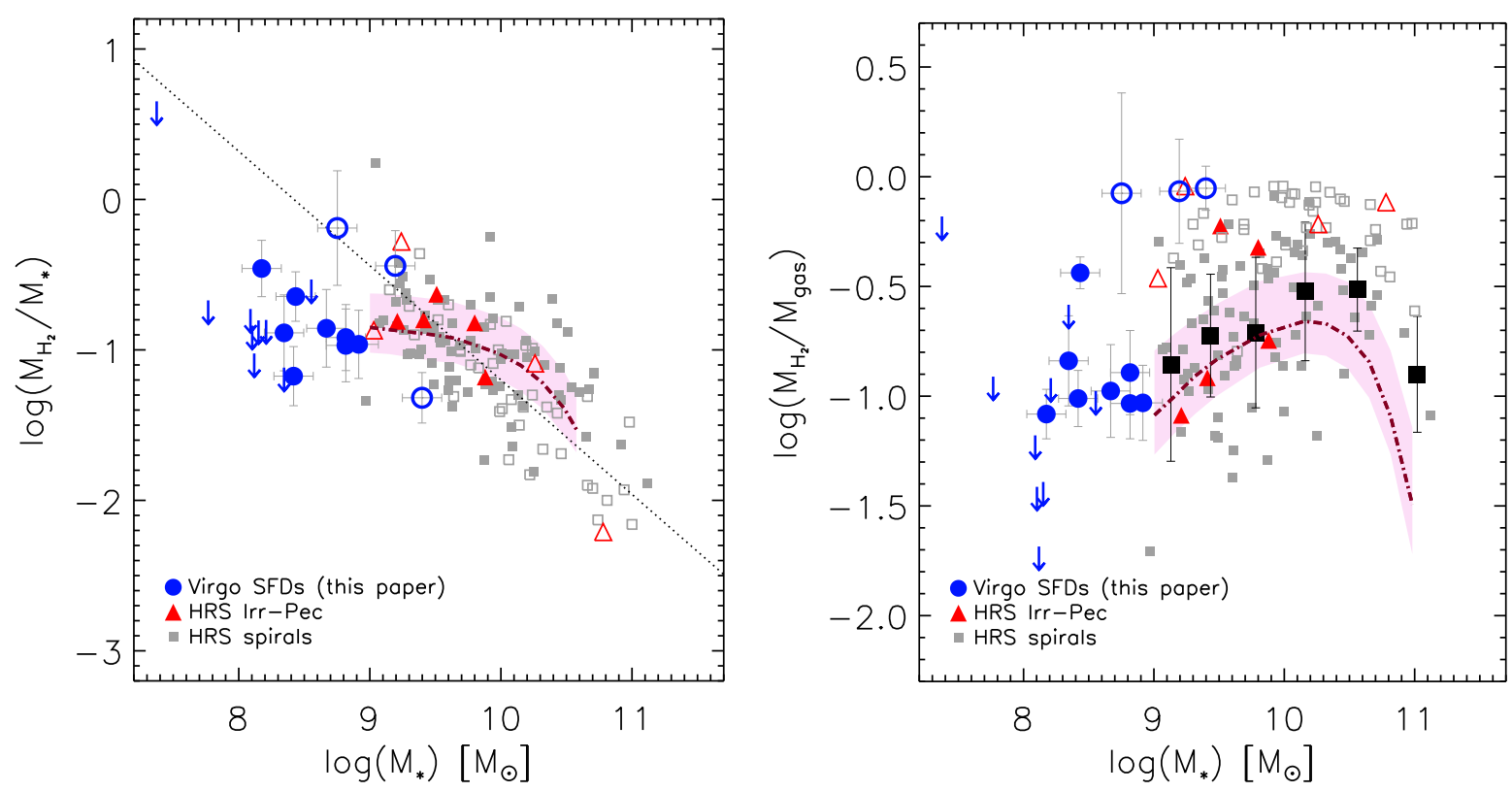

Fig. 5. Left panel: ratio of $\mathrm{H}_{2}$ to stellar mass against stellar mass. Virgo SFDs (blue dots) are compared to spiral (grey squares) and irregularpeculiar (red triangles) galaxies from the HRS. HI-deficient galaxies $\left(D e f_{\mathrm{HI}}>0.5\right)$ in both samples are indicated with empty symbols. The dotted line shows the scaling relation obtained for the HRS galaxies with a normal HI content (Boselli et al. 2014c). Right panel: molecular gas mass fraction against stellar mass. Symbols are the same as in the left panel. Large black squares show the average distribution of the HRS galaxies with a normal HI content. The dot-dashed lines in both panels indicate the predictions of the model of Lu et al. (2015), and the shaded area corresponds to the uncertainty on the model.

by a factor of 9 at most (for the lowest metallicity object of the sample, VCC324), compared to the Milky Way conversion. The $H$-luminosity-dependent and the metallicity-dependent estimates agree reasonably well (within the uncertainties), and the larger discrepancy between the two methods (a factor of $\sim 3$ ) is found for VCC324.

The $\mathrm{H}_{2}$ masses estimated with all the methods discussed in this section are displayed in Table 3. In the rest of the paper we adopt the $H$-luminosity-based estimate because it provides more CO-detected galaxies in the HRS sample. The choice of this $X$ factor does not remarkably modify the main results of our work.

\section{Molecular hydrogen properties of Virgo SFDs}

\subsection{Molecular-to-stellar mass ratio}

To investigate the relation between the stellar and the cold gas mass content of the Virgo SFDs, we display in Fig. 5 (left panel) the ratio of $\mathrm{H}_{2}$ to stellar mass $\left(M_{\mathrm{H}_{2}} / M_{*}\right)$ versus stellar mass. This scaling relation has been thoroughly studied by other molecular gas surveys (Saintonge et al. 2011a; Boselli et al. 2014c). To inspect a broader dynamical range than that sampled by our set of dwarfs, we compared Virgo SFDs to HRS galaxies. To make the samples as homogeneous as possible, we recalculated the stellar masses of Virgo SFDs following the method of Zibetti et al. (2009) that was adopted by the HRS team (Cortese et al. 2012a), based on $g$ and $i$ photometry (see Table A.3). $\mathrm{H}_{2}$ masses in the figure are derived with a $H$-luminosity dependent $X$ factor. In the figure we distinguish between HI-normal $\left(D e f_{\mathrm{HI}} \leq 0.5\right)$ and HI-deficient $\left(D e f_{\mathrm{HI}}>0.5\right)$ galaxies (filled and empty symbols, respectively). The dotted line indicates the anti-correlation between $M_{\mathrm{H}_{2}} / M_{*}$ and stellar mass found in the HRS sample, considering only galaxies with a normal HI content (Boselli et al. 2014c). Virgo dwarfs with $D e f_{\mathrm{HI}} \leq 0.5$ and stellar masses below $10^{9} M_{\odot}$ (filled circles) do not follow the scaling relation
Table 3. Total $\mathrm{H}_{2}$ masses of the Virgo SFD galaxies.

\begin{tabular}{lccc}
\hline \hline ID & $\begin{array}{c}\log M_{\mathrm{H}_{2}}^{\text {gal }} \\
{\left[M_{\odot}\right]}\end{array}$ & $\begin{array}{c}\log M_{\mathrm{H}_{2}}^{L_{H}} \\
{\left[M_{\odot}\right]}\end{array}$ & $\begin{array}{c}\log M_{\mathrm{H}_{2}}^{\mathrm{W} 10} \\
{\left[M_{\odot}\right]}\end{array}$ \\
\hline 10 & $7.41 \pm 0.22$ & $7.84 \pm 0.22$ & $7.54 \pm 0.22$ \\
87 & $<6.98$ & $<7.67$ & $<7.63$ \\
135 & $7.86 \pm 0.08$ & $8.08 \pm 0.08$ & $7.90 \pm 0.08$ \\
144 & $7.31 \pm 0.17$ & $7.77 \pm 0.17$ & $8.06 \pm 0.17$ \\
172 & $<7.88$ & $<8.27$ & $<7.98$ \\
213 & $8.13 \pm 0.35$ & $8.56 \pm 0.35$ & $8.08 \pm 0.35$ \\
324 & $7.09 \pm 0.27$ & $7.60 \pm 0.26$ & $8.05 \pm 0.27$ \\
334 & $<6.51$ & $<7.21$ & $<7.23$ \\
340 & $7.56 \pm 0.17$ & $7.91 \pm 0.17$ & $8.19 \pm 0.17$ \\
562 & $<7.08$ & $<8.15$ & $<8.17$ \\
693 & $<7.12$ & $<7.41$ & $<7.42$ \\
699 & $7.65 \pm 0.17$ & $7.95 \pm 0.17$ & $8.18 \pm 0.17$ \\
737 & $<6.82$ & $<7.45$ & $<7.40$ \\
841 & $<6.68$ & $<7.36$ & $<7.15$ \\
1437 & $7.26 \pm 0.14$ & $7.82 \pm 0.14$ & $7.63 \pm 0.14$ \\
1575 & $8.43 \pm 0.18$ & $8.75 \pm 0.18$ & $8.39 \pm 0.18$ \\
1686 & $7.49 \pm 0.19$ & $7.85 \pm 0.19$ & $7.94 \pm 0.19$ \\
1699 & $<6.87$ & $<7.44$ & $<8.08$ \\
1725 & $7.03 \pm 0.18$ & $7.61 \pm 0.18$ & $7.68 \pm 0.18$ \\
1791 & $<6.98$ & $<7.62$ & $<7.88$ \\
\hline & & &
\end{tabular}

determined in the HRS sample. The trend given by the upper limits also indicates that this deviation extends to the lowest stellar masses sampled by our survey.

The flattening of the molecular-to-stellar mass ratio at low $M_{*}$ is expected by evolution models of the gaseous content of galaxies. The dash-dotted line shows the predictions of the model of Krumholz et al. (2009) derived in Lu et al. (2015), with the shaded area indicating the corresponding uncertainty. 
M. Grossi et al.: Star-forming dwarf galaxies in the Virgo cluster: the link between molecular gas, atomic gas, and dust
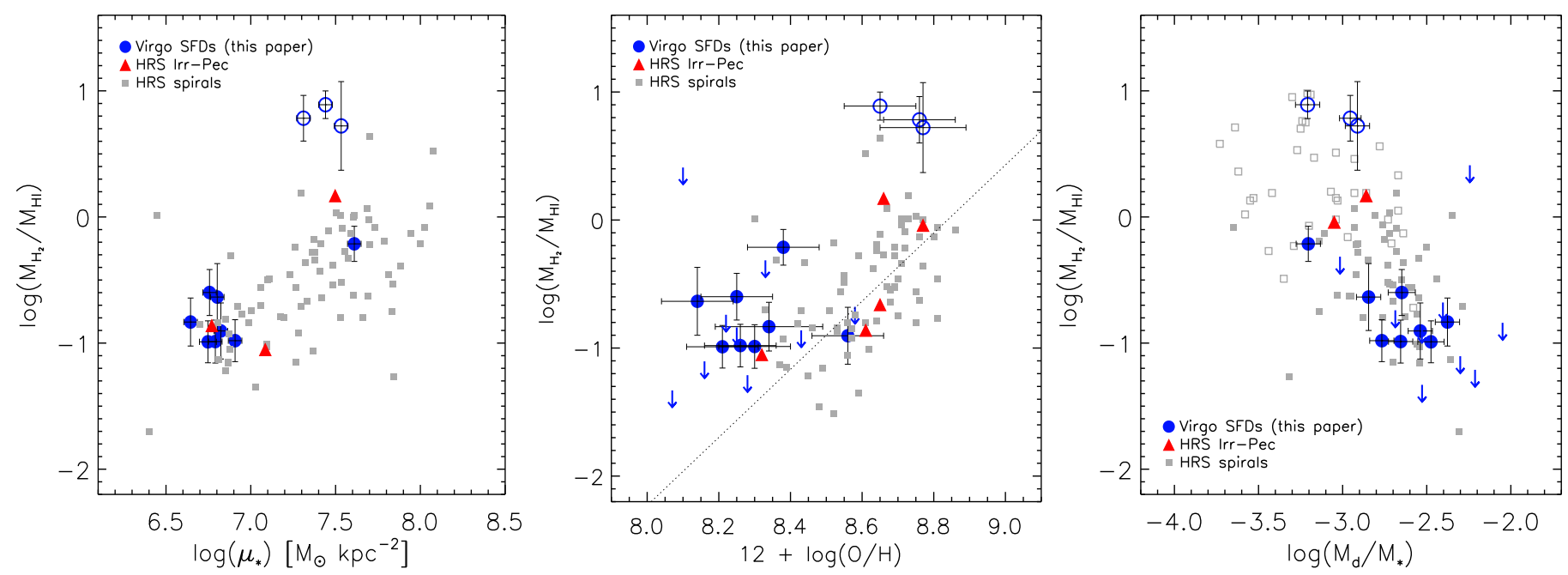

Fig. 6. $\mathrm{H}_{2}$-to-HI ratio against stellar mass surface density (left panel), metallicity (central panel), and ratio of dust to stellar mass (right panel). Virgo SFDs (blue dots) are compared to spiral (grey squares) and irregular-peculiar (red triangles) galaxies from the HRS. HI-deficient galaxies in all samples are indicated with empty symbols. The dotted line in the central panel shows the scaling relation obtained for the HRS galaxies with a normal HI content (Boselli et al. 2014c).

The flattening of the relation can be explained by the role played by metallicity in Krumholz et al. (2009). The model assumes a critical surface density for molecule formation, which is roughly inversely proportional to the gas-phase metallicity. The lower metallicity in galaxies of lower stellar masses affects the process of molecular hydrogen formation, but stars can form in HI-dominated regions, which are very poor in molecules (Glover \& Clark 2012; Krumholz 2013). Therefore, according to this model, the flattening of $M_{\mathrm{H}_{2}} / M_{*}$ at low masses is not the consequence of gas removal due to environmental effects. Of the most HI-deficient galaxies, only VCC135 has a low $\mathrm{H}_{2}$ fraction compared to galaxies with a similar stellar mass, while VCC213 and VCC1575 appear to have a similar $\mathrm{H}_{2}$ content as the other dwarfs.

To verify whether HI dominates the total gas budget at low masses, we show in the right panel of Fig. 5 the scaling relation for the ratio of molecular-to-total gas mass compared to the predictions of Lu et al. (2015, dot-dashed line). The molecular gas fraction of HI-normal SFDs $\left(D e f_{\mathrm{HI}} \leq 0.5\right)$ ranges between $9 \%$ and $38 \%$ of the total amount of gas (with a mean value of $14 \%$ ), and the majority of these dwarfs have $M_{\mathrm{H}_{2}} / M_{\text {gas }}$ ratios comparable to the predictions of the model (extrapolated to $\left.M_{*}<10^{9} M_{\odot}\right)$. The molecular-to-total gas mass ratio is higher in the HI-deficient galaxies (empty circles and squares), and it does not seem to vary remarkably with $M_{*}$ in these systems at low and high stellar masses. The HRS galaxies with a normal gas content show significant scatter, although if we consider the mean values of the sample in bins of stellar masses (large filled squares), they appear to follow the general trend defined by the model. The main contribution of $\mathrm{H}_{2}$ to the total gas mass occurs at stellar masses around $10^{10} M_{\odot}$.

\subsection{Ratio of molecular to atomic gas}

The molecular and atomic gas masses ratio, $R_{\mathrm{mol}}=M_{\mathrm{H}_{2}} / M_{\mathrm{HI}}$ provides information about the conversion of atomic into molecular gas and about the balance between $\mathrm{H}_{2}$ formation and destruction. Models of the $\mathrm{HI}$ to $\mathrm{H}_{2}$ transition (Elmegreen 1993) showed that in regions with low molecular fractions $R_{\mathrm{mol}} \sim P^{2.2} / j$, where $P$ is the interstellar mid-plane hydrostatic gas pressure and $j$ is the mean interstellar radiation field. Wong \& Blitz (2002) and Blitz \& Rosolowsky (2004) also claimed that the interstellar gas pressure plays the dominant role in determining the balance between $\mathrm{HI}$ and $\mathrm{H}_{2}$. However, Krumholz et al. (2009) predicted that the $\mathrm{H}_{2}$-to-HI ratio mainly depends on the total gas column density of a galaxy and only secondarily on the metallicity. It is therefore important to address through observations how $R_{\text {mol }}$ is related to global galaxy properties to better understand how the conversion of atomic into molecular gas is regulated in galaxies, especially in low-mass star-forming systems.

In Fig. 6 we plot $R_{\mathrm{mol}}$ against some of the main global properties of our sample: stellar surface density and metallicity. While Virgo dwarfs appear to follow the correlation between this ratio and stellar surface density (left panel) defined by the locus of the HRS galaxies, the dependence of the molecular-to-atomic ratio on metallicity is less clear at low stellar masses (central panel). The dwarf galaxies with an apparently normal HI content show higher $R_{\text {mol }}$ ratios than the scaling relation obtained by Boselli et al. (2014c) for more massive systems.

The gas content of HI-deficient galaxies (i.e. VCC135, VCC213, and VCC1575; empty circles in Fig. 6) is dominated by molecular hydrogen because all have $R_{\text {mol }}>1$. The survival of molecular gas in the central regions of a HI-stripped dwarf irregular stellar disc could be a relevant step for the formation of nucleated dwarf elliptical galaxies (dEs), given that many cluster dEs have distinct nuclear blue regions (Lisker et al. 2006). Thus these galaxies could provide hints on the morphological transformation of star-forming dwarf galaxies into early-type systems in a cluster environment. VCC135 shows a blue core with strong emission lines overlaid on an extended redder stellar structure with an elliptical symmetry; VCC213, on the other hand, has a red core that resembles the nucleus of dwarf elliptical, and star formation is occurring in a ringed or spiral structure around the core (Meyer et al. 2014). Both have global optical colours redder than other BCDs in Virgo ( $g-i>0.7$; Meyer et al. 2014). The redder colours, the structural properties and visual appearance, and the low HI content provide clear indication of the different evolutionary stage of these galaxies that is probably due to the influence of the environment. 
Lastly, in the right panel of Fig. 6 we show how the $\mathrm{H}_{2}$-to-HI ratio varies with the dust fraction $\left(M_{\mathrm{d}} / M_{*}\right)$. Once again, Virgo SFDs are compared to the HRS late-type galaxies. ${ }^{12}$ The figure shows that the ratio is inversely correlated with $R_{\mathrm{mol}}$ and that the same trend holds for both HI-deficient and HI-normal galaxies. This might be related to the fact that HI-poor galaxies are being stripped of their dust content, revealing a dust deficiency as shown by previous Herschel surveys (Cortese et al. 2010, 2012b; Corbelli et al. 2012; Grossi et al. 2015). In Sect. 7.5 we discuss the effects of the environment on the dust and molecular gas components of our sample of dwarfs and we compare them to the galaxies of the HRS.

\subsection{Dust-to-gas mass ratio}

The dust-to-gas mass ratio $\mathcal{D}\left(M_{\mathrm{d}} / M_{\mathrm{g}}\right)$ gives an indication of the enrichment of the gas by heavy elements produced in stars $(\mathrm{C}, \mathrm{O}, \mathrm{Mg}, \mathrm{Si}, \mathrm{Fe})$, the amount of metals that are locked in dust grains, and the net balance between the production and growth of dust grains and their destruction in the ISM. If the ratio of dust-to-metals in the ISM does not vary among galaxies, the relation between $\mathcal{D}$ and the oxygen abundance $\mathrm{O} / \mathrm{H}$ is expected to be linear (Edmunds 2001). Previous studies have shown that indeed there is a nearly linear relation between $\mathcal{D}$ and the metallicity at oxygen abundances $12+\log (\mathrm{O} / \mathrm{H}) \gtrsim 8.1$ (Draine et al. 2007; Leroy et al. 2011; Sandstrom et al. 2013), while the non-linear behavior becomes notable for metallicities below this value (Rémy-Ruyer et al. 2014).

In Fig. 7 we explore the variation of $\mathcal{D}$ with nebular oxygen abundance for our sample. In the top panel the gas-to-dust ratio is derived by combining the molecular and total atomic gas masses. $\mathcal{D}$ correlates with metallicity, but most of the Virgo dwarfs, especially the more gas-rich ones, have dust-to-gas ratios lower than expected by linearly scaling the Milky Way values (Fig. 7, top panel):

$\mathcal{D}=\frac{M_{\mathrm{d}}}{M_{\mathrm{HI}}+M_{\mathrm{H}_{2}}}=0.01 \frac{(\mathrm{O} / \mathrm{H})}{(\mathrm{O} / \mathrm{H})_{\odot}}$,

assuming a Milky Way dust-to-gas ratio of $\approx 0.01$ (Draine 2011) and oxygen abundance $12+\log (\mathrm{O} / \mathrm{H})=8.69$ (Asplund et al. $2009)^{13}$. As we discussed in Sect. 7.2, our dust mass estimates based on an MMB fit with $\beta=1.5$ are lower by $\sim 0.1$ dex than the most commonly adopted emissivity index $\beta=2$. Despite this variation, as a result of the choice of $\beta$, an average difference of 0.1 dex would still be too low to explain the discrepancy between

12 Dust masses for the HRS have been calculated in Ciesla et al. (2014) by fitting models of Draine \& Li (2007) and in Cortese et al. (2014) using an MBB with a fixed emissivity $\left(\beta=2\right.$ and $\kappa_{0}=3.76 \mathrm{~cm}^{2} \mathrm{~g}^{-1}$ at $\lambda=250 \mu \mathrm{m}$ ). However, to avoid biases when we compare dust masses of the two samples (HRS and Virgo SFDs), we decided to recalculate $M_{\mathrm{d}}$ for the HRS following the same method as adopted in Grossi et al. (2015). We fitted the SED between 100 and $350 \mu \mathrm{m}$ only (because of the $500 \mu \mathrm{m}$ excess detected in most of the Virgo dwarfs) with a single MBB with fixed emissivity $(\beta=1.5)$ and $\kappa_{0}=3.4 \mathrm{~cm}^{2} \mathrm{~g}^{-1}$ at $\lambda=$ $250 \mu \mathrm{m}$. The resulting measurements, displayed in the right panel of Fig. 6 and the bottom left panel of Fig. 9, are on average lower by a factor $0.08 \pm 0.1 \mathrm{dex}$ than the dust masses in Cortese et al. (2014) for $\beta=2$. However, the choice of $\beta$ and $\kappa_{0}$ to estimate dust masses does not affect the conclusions drawn from these figures because the same trends hold if we use either our method or the approach of Cortese et al. (2014) for both samples.

${ }^{13}$ Nonetheless, we are aware that a wide spread of $\mathcal{D}$ values is observed in the linear regime related to different galactic star formation histories (Sandstrom et al. 2013; Rémy-Ruyer et al. 2014; Zhukovska 2014).

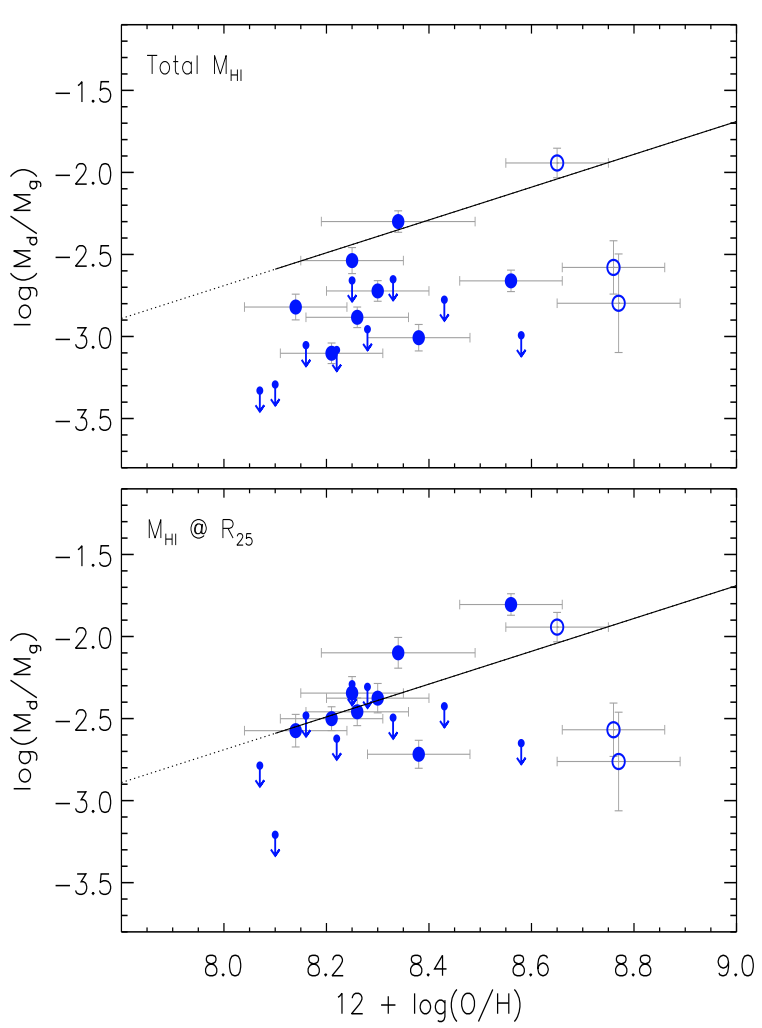

Fig. 7. Top-panel: dust-to-gas mass ratios against metallicity for Virgo SFDs. The solid line indicates a linear scaling of the Milky Way dust-togas ratio and metallicity which holds for galaxies with $12+\log (\mathrm{O} / \mathrm{H}) \gtrsim$ 8.1. The total gas mass here is calculated considering the total HI mass from ALFALFA observations. Bottom-panel: same as upper panel. The total gas mass here is calculated considering only the HI mass within $R_{25}$, as explained in Sect. 7.3.

the linear scaling of the Milky Way and the measured $\mathcal{D}$ of our dwarfs.

A possible explanation could be that the HI masses from the ALFALFA catalogue only provide the global HI content because our dwarfs are unresolved by the $\sim 3$ '5 Arecibo beam. HI envelopes in dwarf galaxies are known to be remarkably more extended than the stellar (and dust) component (Swaters et al. 2002). $\mathcal{D}$ might therefore be underestimated in some cases because of the different size of the atomic gas disc compared to the extension of the dust and molecular gas reservoir $\left(\lessgtr R_{25}\right)$, but also because of dust and gas stripping as galaxies move through the intracluster medium. The dwarfs with the highest metallicities in our sample are also the most HI-deficient, and we have shown in Grossi et al. (2015) that such galaxies show evidence of dust stripping as well.

To fairly estimate $\mathcal{D}$, it is important that we consider only the HI mass within the extension of the dust disc, which we consider to be equal to $R_{25}$ (Hunt et al. 2015a). However, without high-resolution maps of the neutral hydrogen distribution, we can only derive an approximate estimate of this value. To this aim we assumed that the HI surface density profile follows an exponential law, $\Sigma_{\mathrm{HI}}(r)=\Sigma_{\mathrm{HI}}(0) \mathrm{e}^{-r / h}$, where $\Sigma_{\mathrm{HI}}(0)$ is the central HI surface density and $h$ the HI disc scale length (van Zee et al. 2001; Swaters et al. 2002). From the total HI mass measured with ALFALFA we derived $h$, assuming $\Sigma_{\mathrm{HI}}(0)=6 M_{\odot} \mathrm{pc}^{-2}$, the average central HI surface density observed by Swaters et al. (2002) for a sample of 73 late-type dwarf galaxies. This value 

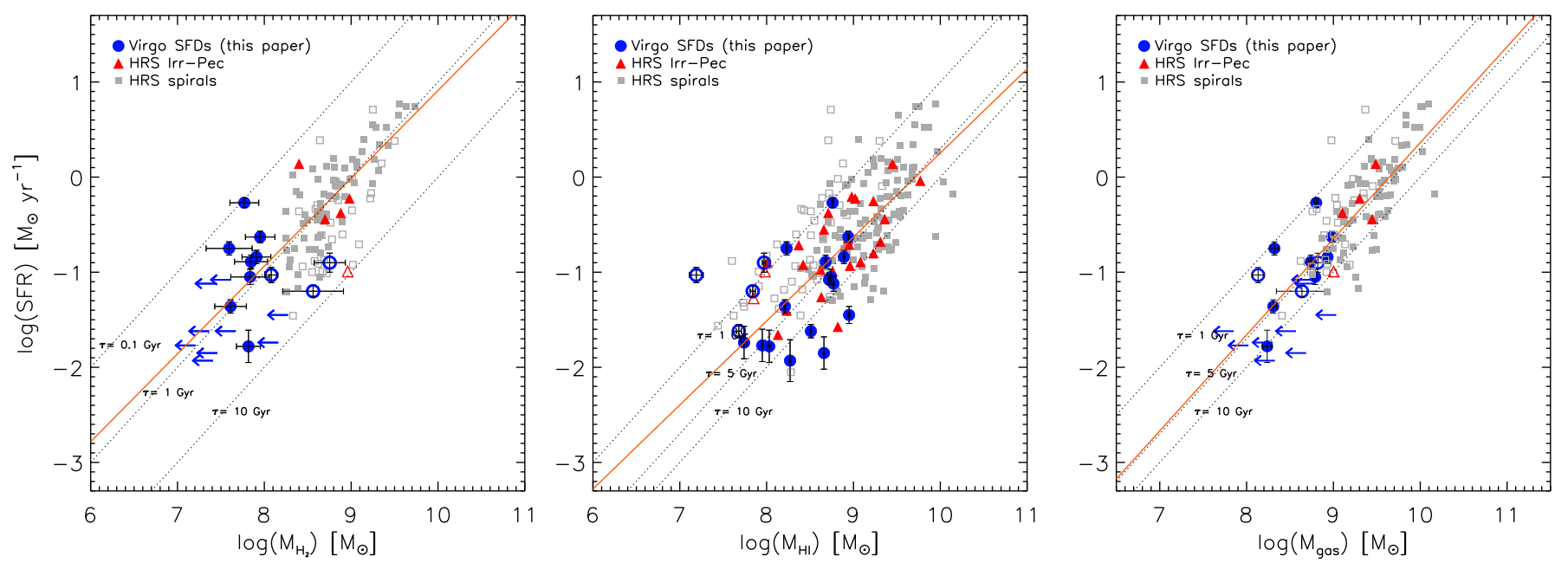

Fig. 8. Left panel: star formation rate against $\mathrm{H}_{2}$ mass. Virgo SFDs (blue dots) are compared to the HRS spiral (grey squares) and irregularpeculiar (red triangles) galaxies. HI-deficient galaxies are indicated with empty symbols, and blue arrows are Virgo dwarf upper limits. The solid line shows the best-fit relation. Dotted lines indicate constant molecular gas depletion timescales of 0.1, 1, and 10 Gyr. Central panel: star formation rate against HI mass. Symbols are the same as in the left panel. The solid line shows the best-fit relation. Dotted lines indicate constant atomic gas depletion timescales of 1,5 , and $10 \mathrm{Gyr}$. Right panel: star formation rate against total gas mass $\left(M_{\mathrm{H}_{2}}+M_{\mathrm{HI}}\right)$. Symbols are the same as in the left panel. The solid line shows the best-fit relation. Total gas depletion timescales of 1,5 , and $10 \mathrm{Gyr}$ are also overlaid. Coefficients of the best-fit relations are given in Table 4.

is also compatible with those obtained from VLA observations of a few of the Virgo dwarfs included in our sample (Hoffman et al. 2003). Then we calculated $\Sigma_{\mathrm{HI}}\left(R_{25}\right)$ and the corresponding HI mass. The uncertainty on the masses was estimated assuming that $\Sigma_{\mathrm{HI}}(0)$ varies between 4 and $8 M_{\odot} \mathrm{pc}^{-2}$, the $1 \sigma$ dispersion determined in the sample of Swaters et al. (2002).

In the lower panel of Fig. 7, we show the same plot as in the upper panel, with HI masses calculated within the optical radius $R_{25}$. The figure shows that when we follow this approach, most of the dwarfs with a normal HI content lie along the linear relation defined by Eq. (17). This confirms the importance of calculating the total gas and dust masses within the same region to prevent biases in the estimate of $\mathcal{D}$, as has also been stressed by previous works (Draine et al. 2007; Herrera-Camus et al. 2012; Rémy-Ruyer et al. 2014).

\subsection{Integrated star formation laws}

Molecular gas and star formation show an extremely tight correlation across a wide range of galaxy types, both in the local Universe and at high redshift, through the Kennicutt-Schmidt relation (Schmidt 1959; Kennicutt 1989, 1998b). The slope of the power-law relation and the rank correlation depend on the spatial resolution sampled by the data (Bigiel et al. 2008), the type of galaxy (star-forming, starburst), the examined gas component $\left(\mathrm{H}_{2}, \mathrm{HI}\right.$, or total gas), and the local environment (inner or outer disc; Bigiel et al. 2008; Bolatto et al. 2011; Leroy et al. 2011; Schruba et al. 2011).

Studying the star formation law in dwarf galaxies allows us to probe a different physical regime of the ISM compared to spirals because the dominant gas component is neutral hydrogen on a large scale, and surface gas densities and metal abundances are lower (Hunter 1997; Hunter et al. 2011; Schruba et al. 2012), similarly to the outer region of spiral discs (Elmegreen \& Hunter 2015).

We only had integrated measurements available (total $\mathrm{HI}, \mathrm{H}_{2}$ and total SFRs), therefore we examine in Fig. 8 the integrated
Table 4. Best-fit coefficients of the integrated star formation laws: log $(S F R)=a x+b$.

\begin{tabular}{ccc}
\hline \hline$x$ & $a$ & $b$ \\
\hline $\log \left(M_{\mathrm{H}_{2}}\right)$ & $0.92 \pm 0.07$ & $-8.32 \pm 0.22$ \\
$\log \left(M_{\mathrm{H}_{I}}\right)$ & $0.88 \pm 0.07$ & $-8.58 \pm 0.22$ \\
$\log \left(M_{\text {gas }}\right)$ & $1.01 \pm 0.08$ & $-9.74 \pm 0.24$ \\
\hline
\end{tabular}

star formation laws for the different gas components: molecular, atomic, and total gas. Again we compared Virgo dwarfs to the HRS sample ${ }^{14}$ - covering approximately three orders of magnitude in gas masses and SFRs. A linear fit to the data points (including only galaxies with a normal HI content) is consistent with a linear relation for the molecular and total components (left and right panels of Fig. 8, orange lines), with a slope of 0.93 and 1.01, respectively (Table 4). For the atomic gas component the slope is slightly lower, 0.88 , indicating that overall, molecular gas is better correlated with star formation than HI (see Table 4).

The ratio of the molecular mass to the SFR, $\tau_{\text {dep }}=M_{\mathrm{H}_{2}} / S F R$, measures the molecular gas depletion timescale, that is, the amount of time for which a galaxy can sustain star formation at the current rate without accreting gas from the external environment (closed-box model). The inverse of this parameter gives the SFR per unit of molecular gas, or the efficiency at transforming $\mathrm{H}_{2}$ into stars. Dotted lines show constant molecular gas depletion timescales of $0.1,1$, and $10 \mathrm{Gyr}$, with the main locus of the distribution lying at $\tau=M_{\mathrm{H}_{2}} / S F R=1$ Gyr. Resolved studies of the discs of nearby spiral galaxies on kpc scales have shown that $\tau_{\text {dep }}$ is roughly constant at $\approx 2$ Gyr (Bigiel et al. 2008; Leroy et al. 2013). However, systematic variations of the $\mathrm{H}_{2}$ depletion timescale are found when $\tau_{\text {dep }}$ is considered to be averaged over the entire galaxy instead of spatially resolved maps

${ }^{14}$ SFRs for the HRS survey have been taken from Boselli et al. (2015) and converted into a Kroupa IMF. They were derived using the same calibration $(\mathrm{H} \alpha+22 \mu \mathrm{m})$ as was applied to our sample. 

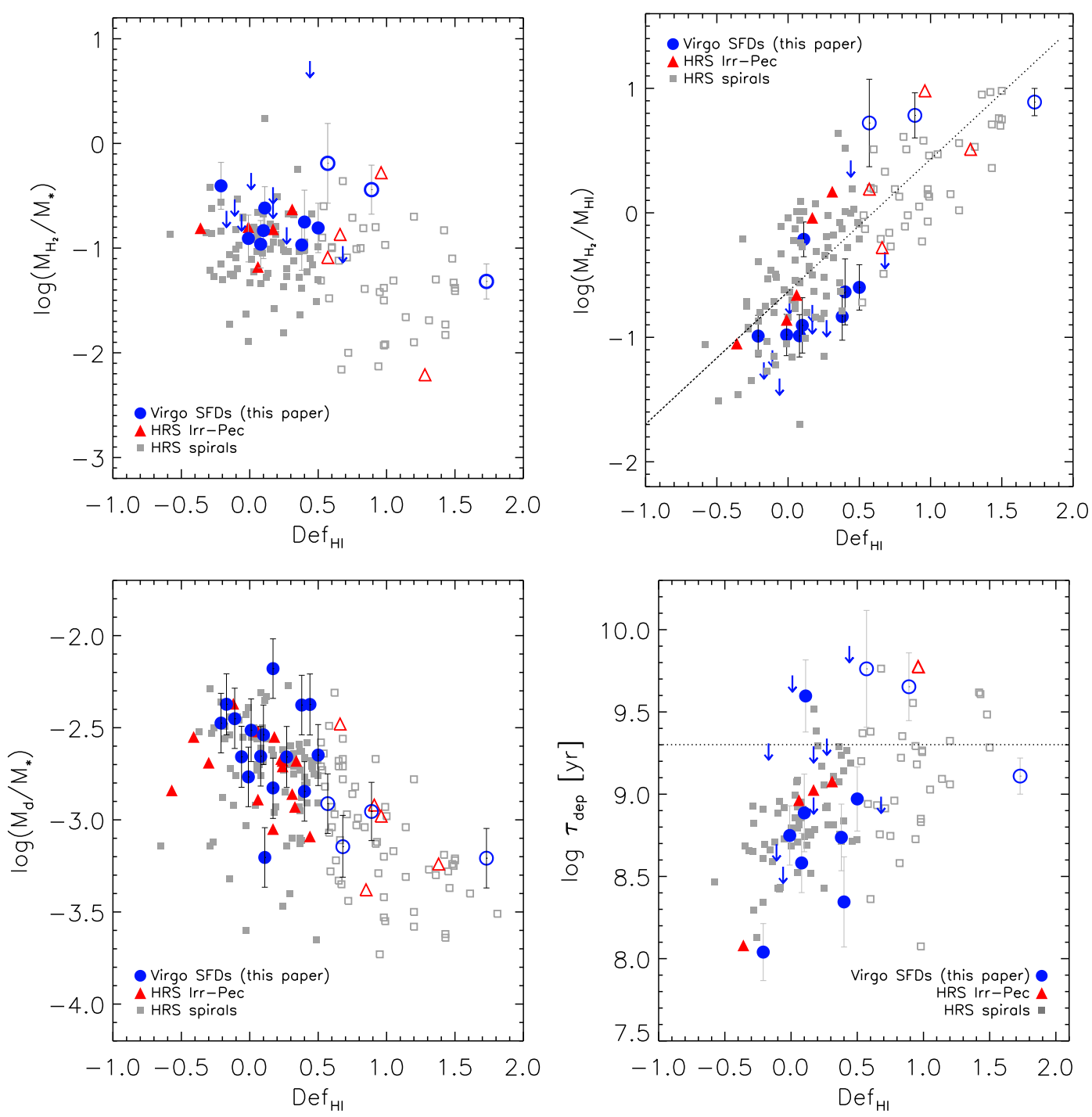

Fig. 9. Environmental effects on the different ISM components. Top left: ratio of $\mathrm{H}_{2}$ to stellar mass against $\mathrm{HI}$ deficiency. Top right: ratio of $\mathrm{H}_{2}$ to HI mass against HI deficiency. Bottom-left: ratio of dust to stellar mass against HI deficiency. Bottom right: molecular gas depletion timescale against HI deficiency. Symbols are the same as in Fig. 5.

(Saintonge et al. 2011b; Leroy et al. 2013; Boselli et al. 2014c; Hunt et al. 2015b). Low-mass, low-metallicity, and gas-rich systems are on average more efficient at producing stars than normal spiral discs, and trends are observed between $\tau_{\text {dep }}$ and stellar mass (Leroy et al. 2013), specific SFR (Hunt et al. 2015b), and metallicity (Boselli et al. 2014c). Molecular gas depletion timescales vary among the Virgo SFDs. The dwarfs with a normal HI content are more efficient at forming stars than normal discs, and five out of ten objects have $\tau_{\text {dep }}$ between $100 \mathrm{Myr}$ and $1 \mathrm{Gyr}$, while all the HI-deficient objects have $\tau_{\text {dep }}$ comparable to or longer than the value found in normal spiral galaxies ( 2-3 Gyr).

VCC1437 is the dwarf with the longest molecular gas consumption rate among the Virgo sample ( $\sim 4.5 \mathrm{Gyr})$. It has a low SFR $\left(\sim 0.02 M_{\odot} \mathrm{yr}^{-1}\right)$, despite its consistent reservoir of both atomic and molecular gas (36\% of the total baryonic mass), thus it is not clear why star formation appears to be very inefficient in this galaxy. SDSS images of VCC1437 shows a spherically symmetric stellar component, with a centrally concentrated region of star formation activity. The galaxy is classified as a nuclear elliptical (nE) BCD (Lisker et al. 2006; Meyer et al. 2014) according to the BCD morphological classification of Loose \& Thuan (1986), meaning that the nuclear star-forming region is overlaid on an old population of low-mass stars with a spherical or elliptical distribution. Thus, it is likely that this dwarf galaxy is in a different evolutionary stage than the other systems, which do not show such a spherical symmetric stellar component and host patchy star formation regions throughout the disc.

\subsection{Environmental effects on the ISM}

From the combined analysis of the dust, atomic, and molecular hydrogen components we can assess how the ISM of Virgo SFDs is perturbed and depleted as they fall into the cluster substructures. In this section we compare IRAM data with Herschel and $21 \mathrm{~cm}$ observations to identify variations in the cold phases of the ISM (and eventually in the star formation activity) according to the stage of interaction with the cluster environment. The different levels of HI deficiency can provide information on the degree of perturbation the galaxies are experiencing. 
In Fig. 9 we display the molecular gas-to-stellar mass ratio (top-left panel), the $\mathrm{H}_{2}$-to-HI ratio (top-right panel), the dust-to-stellar mass ratio (bottom-left panel), and the molecular gas depletion timescale against HI deficiency (bottom right panel). Virgo SFDs are compared to the HRS galaxies. The $M_{\mathrm{H}_{2}} / M_{*}$ ratio of the HI-deficient dwarfs does not remarkably differ from that of the HI-normal ones (top-left panel), suggesting that despite the poor statistics, there is no molecular gas deficiency in the HI-poor dwarfs. A modest variation of $M_{\mathrm{H}_{2}} / M_{\mathrm{HI}}$ can be seen in the HRS sample. Boselli et al. (2014b) claimed that Virgo cluster galaxies have, on average, a lower molecular gas content than similar objects in the field and that $\mathrm{H}_{2}$ is also removed by the cluster environment through ram-pressure stripping, but less efficiently so than the atomic gas. This different efficiency is shown in the top right panel, where we display the variation of $\mathrm{H}_{2} / \mathrm{HI}$ mass ratio with $D e f_{\mathrm{HI}}$. Virgo SFDs with high values of this ratio are the most HI-deficient, similarly to the HRS objects, and most of the galaxies in the plot follow a linear relation (dotted line), which implies a normal $\mathrm{H}_{2}$ content (Kenney \& Young 1986). Both figures suggest that the mechanism removing the low-density atomic gas has left the molecular component mostly intact at the current stage of evolution of the star-forming dwarfs within the cluster, probably because of their different spatial distribution $\left(\mathrm{H}_{2}\right.$ is more centrally concentrated than HI, with a shorter scale length; Leroy et al. 2009b). Highly HI-deficient galaxies are also characterised by lower dust fractions (bottom left panel), which confirms that dust stripping occurs with HI stripping in both low- and high-mass galaxies in the Virgo cluster (Corbelli et al. 2012; Cortese et al. 2012b; Grossi et al. 2015).

The amount of gas available to sustain star formation depends on various factors, including the inflow of gas from the external environment, the re-accretion of ejected gas, and the availability of large gas reservoirs (extended HI discs). If all these factors are affected by the interaction with the cluster environment, a reduced star formation activity as well as longer gas depletion timescales might be expected. In the bottom right panel of Fig. 9 we plot $\tau_{\text {dep }}$ against HI deficiency. The two parameters appear correlated on a statistical basis (Spearman rank correlation coefficient $r_{\mathrm{s}}=0.57$, two-sided significance of its deviation from zero $p=4.8 \times 10^{-11}$ ), and the most HI-deficient galaxies show a less efficient star formation activity. Both samples show that the molecular gas depletion timescales increase with the HI deficiency. The 2 Gyr value determined in the HERACLES sample (Bigiel et al. 2008) is indicated by the dotted line in the figure. Because the greatest HI depletion so far has occurred mainly in the outer discs, outside the region in which most of the molecular gas is located, this plot seems to suggest that star formation in the HI-deficient dwarfs is slowing down because the gas supply is not replenished from the outer regions of the disc.

\section{Summary and conclusions}

We have presented IRAM 30-m telescope observations at 115 and $230 \mathrm{GHz}$ of a sample of 20 Virgo cluster SFD galaxies. The dwarfs, selected from the Herschel Virgo Cluster Survey, have oxygen abundances within the range $8.1 \lesssim 12+$ $\log (\mathrm{O} / \mathrm{H}) \lesssim 8.8 .{ }^{12} \mathrm{CO}(1-0)$ and ${ }^{12} \mathrm{CO}(2-1)$ emission is detected in 11 out of 20 objects down to an oxygen abundance of $12+\log (\mathrm{O} / \mathrm{H})=8.1$, including a tentative $\simeq 3 \sigma$ detection of VCC1725 at $115 \mathrm{GHz}$. The most significant difference between detections and non-detections is the higher stellar mass, and, consequently, the higher SFR.
We corrected $\mathrm{CO}$ fluxes taking into account both aperture corrections (when the $\mathrm{CO}$ emission was more extended than the IRAM 30-m telescope beam size) and the finite source size (if the emitting region is smaller than the beam). CO fluxes correlate with FIR $250 \mu \mathrm{m}$ emission with a slope that is close to linear. The correlation holds from Virgo spirals to the dwarf sample and covers three orders of magnitude in both $\mathrm{CO}$ fluxes and $250 \mu \mathrm{m}$ flux densities. The link between cold dust and molecular gas appears to be stronger than that with warm dust traced by MIR emission.

$\mathrm{H}_{2}$ masses were derived using a $H$-luminosity-dependent factor (Boselli et al. 2002) and a metallicity-dependent CO-to$\mathrm{H}_{2}$ conversion factor (Wolfire et al. 2010), and we showed that both methods lead to comparable mass estimates. The ratio of molecular to stellar mass of Virgo SFDs with a normal HI content remains nearly unchanged below $M_{*} \sim 10^{9} M_{\odot}$, contrary to the $\mathrm{HI}$ fraction, $M_{\mathrm{HI}} / M_{*}$, which increases inversely with $M_{*}$ (Grossi et al. 2015). The total gaseous budget is dominated by the atomic hydrogen component in SFDs, which have a normal HI content. The mean molecular hydrogen content is $\sim 14 \%$ of the total gas, compared to $25-30 \%$ in spiral galaxies (Boselli et al. 2014c). On the other hand, $\mathrm{H}_{2}$ is the main gaseous component in HI-poor systems. The molecular-to-atomic ratio is better correlated with stellar surface density than metallicity, confirming that the interstellar gas pressure plays a key role in determining the balance between the two gaseous components of the ISM.

Virgo dwarfs follow the same linear trend between molecular, atomic, and total gas mass and SFR of more massive spirals, but the correlation and the scatter around it is tighter for molecular and total gas than for atomic hydrogen. Molecular gas depletion timescales, $\tau_{\text {dep }}$, vary between $100 \mathrm{Myr}$ and 6 Gyr, with one galaxy, VCC1437, showing a remarkably low star formation efficiency despite its consistent reservoir of both $\mathrm{HI}$ and $\mathrm{H}_{2}$.

The interaction with the cluster environment is stripping the low-density atomic gas and dust, while the more centrally concentrated molecular gas component appears to be mostly intact at the current stage of evolution of the dwarfs. We did not find any particular difference in the ratio of molecular to stellar mass of HI-normal or HI-deficient galaxies, contrary to the other components of the ISM. The correlation between HI deficiency and $\tau_{\text {dep }}$ suggests that the lack of gas replenishment from the outer regions of the disc also lowers the star formation activity. As the galaxies enters the denser regions of the cluster, their ISM will be removed by ram-pressure stripping, and star formation will be gradually quenched. They will evolve into quiescent galaxies, but they might be able to conserve, at least on short timescales, their angular momentum and rotation (Toloba et al. 2009). Thus, our sample may give hints about the precursors of rotation-supported early-type dwarfs, with signs of discs and blue nuclei, which are usually found at larger distances to the core of the Virgo cluster (Lisker et al. 2006; Toloba et al. 2009; Meyer et al. 2014).

Acknowledgements. We thank the anonymous referee for the constructive and timely comments that helped us to improve the manuscript. M.G. gratefully acknowledges support from CAPES (through grant "A formação de galáxias starburst: histórico dos últimos 10 bilhões de anos", Call n. 001/2010). I.D.L. gratefully acknowledges the support of the Flemish Fund for Scientific Research (FWO-Vlaanderen). L.K.H. acknowledges support from PRIN-INAF 2012/13. We are grateful to the IRAM 30-m staff for their support during the observations. We thank Sandra Treviño-Morales for helping us to retrieve observation files from the IRAM archive. This work has benefited from research funding from the European Community's Seventh Framework Programme. M.G., L.B., and E.L. acknowledge travel support to Pico Veleta from TNA Radio Net project funded 
by the European Commission within the FP7 Programme. The research leading to these results has received funding from the European Commission Seventh Framework Programme (FP/2007-2013) under grant agreement No. 283393 (RadioNet3).

\section{References}

Albrecht, M., Chini, R., Krügel, E., Müller, S. A. H., \& Lemke, R. 2004, A\&A, 414,141

Asplund, M., Grevesse, N., Sauval, A. J., \& Scott, P. 2009, ARA\&A, 47, 481

Auld, R., Bianchi, S., Smith, M. W. L., et al. 2013, MNRAS, 428, 1880

Bell, M. B. 1993, ApJ, 417, 305

Bianchi, S. 2013, A\&A, 552, A89

Bigiel, F., Leroy, A., Walter, F., et al. 2008, AJ, 136, 2846

Binggeli, B., Sandage, A., \& Tammann, G. A. 1985, AJ, 90, 1681

Binggeli, B., Tammann, G. A., \& Sandage, A. 1987, AJ, 94, 251

Blitz, L., \& Rosolowsky, E. 2004, ApJ, 612, L29

Bohlin, R. C., Savage, B. D., \& Drake, J. F. 1978, ApJ, 224, 132

Boselli, A., \& Gavazzi, G. 2006, PASP, 118, 517

Boselli, A., Lequeux, J., \& Gavazzi, G. 2002, A\&A, 384, 33

Boselli, A., Eales, S., Cortese, L., et al. 2010, PASP, 122, 261

Bolatto, A. D., Leroy, A. K., Jameson, K., et al. 2011, ApJ, 741, 12

Bolatto, A. D., Wolfire, M., \& Leroy, A. K. 2013, ARA\&A, 51, 207

Boselli, A., Cortese, L., \& Boquien, M. 2014a, A\&A, 564, A65

Boselli, A., Cortese, L., Boquien, M., et al. 2014b, A\&A, 564, A67

Boselli, A., Cortese, L., Boquien, M., et al. 2014c, A\&A, 564, A66

Boselli, A., Voyer, E., Boissier, S., et al. 2014d, A\&A, 570, A69

Boselli, A., Fossati, M., Gavazzi, G., et al. 2015, A\&A, 579, A102

Bot, C., Boulanger, F., Rubio, M., \& Rantakyro, F. 2007, A\&A, 471, 103

Boulanger, F., Abergel, A., Bernard, J.-P., et al. 1996, A\&A, 312, 256

Brinchmann, J., Charlot, S., White, S. D. M., et al. 2004, MNRAS, 351, 1151

Chang, Y.-Y., van der Wel, A., da Cunha, E., \& Rix, H.-W. 2015, ApJS, 219, 8

Ciesla, L., Boselli, A., Smith, M. W. L., et al. 2012, A\&A, 543, A161

Ciesla, L., Boquien, M., Boselli, A., et al. 2014, A\&A, 565, A128

Corbelli, E., Giovanardi, C., Palla, F., \& Verley, S. 2011, A\&A, 528, A116

Corbelli, E., Bianchi, S., Cortese, L., et al. 2012, A\&A, 542, A32

Cortese, L., Davies, J. I., Pohlen, M., et al. 2010, A\&A, 518, L49

Cortese, L., Catinella, B., Boissier, S., Boselli, A., \& Heinis, S. 2011, MNRAS 415, 1797

Cortese, L., Boissier, S., Boselli, A., et al. 2012a, A\&A, 544, A101

Cortese, L., Ciesla, L., Boselli, A., et al. 2012b, A\&A, 540, A52

Cortese, L., Fritz, J., Bianchi, S., et al. 2014, MNRAS, 440, 942

Dame, T. M., Hartmann, D., \& Thaddeus, P. 2001, ApJ, 547, 792

Davies, J. I., Bianchi, S., Cortese, L., et al. 2012, MNRAS, 419, 3505

Draine, B. T. 2011, Physics of the Interstellar and Intergalactic Medium (Princeton University Press)

Draine, B. T., \& Li, A. 2007, ApJ, 657, 810

Draine, B. T., Dale, D. A., Bendo, G., et al. 2007, ApJ, 663, 866

Edmunds, M. G. 2001, MNRAS, 328, 223

Elmegreen, B. G. 1993, ApJ, 411, 170

Elmegreen, B. G., \& Hunter, D. A. 2015, ApJ, 805, 145

Ftaclas, C., Struble, M. F., \& Fanelli, M. N. 1984, ApJ, 282, 19

Fumagalli, M., Krumholz, M. R., Prochaska, J. X., Gavazzi, G., \& Boselli, A. 2009, ApJ, 697, 1811

Gavazzi, G., Boselli, A., Donati, A., Franzetti, P., \& Scodeggio, M. 2003, A\&A, 400,451

Gavazzi, G., Fumagalli, M., Fossati, M., et al. 2013, A\&A, 553, A89

Gavazzi, G., Franzetti, P., \& Boselli, A. 2014, ArXiv e-prints [arXiv: 1401.8123]

Giovanelli, R., Haynes, M. P., Kent, B. R., et al. 2005, AJ, 130, 2598

Glover, S. C. O., \& Clark, P. C. 2012, MNRAS, 421, 9

Grossi, M., Hunt, L. K., Madden, S. C., et al. 2015, A\&A, 574, A126

Groves, B. A., Schinnerer, E., Leroy, A., et al. 2015, ApJ, 799, 96

Harris, J., \& Zaritsky, D. 2004, AJ, 127, 1531

Haynes, M. P., \& Giovanelli, R. 1984, AJ, 89, 758

Haynes, M. P., Giovanelli, R., Martin, A. M., et al. 2011, AJ, 142, 170

Heeschen, D. S. 1961, ApJ, 133, 322

Herrera-Camus, R., Fisher, D. B., Bolatto, A. D., et al. 2012, ApJ, 752, 112

Hoffman, G. L., Helou, G., Salpeter, E. E., \& Lewis, B. M. 1989, ApJ, 339, 812

Hoffman, G. L., Brosch, N., Salpeter, E. E., \& Carle, N. J. 2003, AJ, 126, 2774
Huang, S., Haynes, M. P., Giovanelli, R., \& Brinchmann, J. 2012, ApJ, 756, 113 Hughes, T. M., Cortese, L., Boselli, A., Gavazzi, G., \& Davies, J. I. 2013, A\&A, 550, A115

Hunt, L. K., Draine, B. T., Bianchi, S., et al. 2015a, A\&A, 576, A33

Hunt, L. K., García-Burillo, S., Casasola, V., et al. 2015b, A\&A, 583, A114

Hunter, D. 1997, PASP, 109, 937

Hunter, D. A., Elmegreen, B. G., Oh, S.-H., et al. 2011, AJ, 142, 121

Israel, F. P. 1997, A\&A, 328, 471

Israel, F. P. 2005, A\&A, 438, 855

Israel, F. P., Johansson, L. E. B., Rubio, M., et al. 2003, A\&A, 406, 817

Kenney, J. D., \& Young, J. S. 1986, ApJ, 301, L13

Kennicutt, Jr., R. C. 1989, ApJ, 344, 685

Kennicutt, Jr., R. C. 1998a, ARA\&A, 36, 189

Kennicutt, Jr., R. C. 1998b, ApJ, 498, 541

Kroupa, P. 2001, MNRAS, 322, 231

Krumholz, M. R. 2013, MNRAS, 436, 2747

Krumholz, M. R., McKee, C. F., \& Tumlinson, J. 2009, ApJ, 693, 216

Kuno, N., Sato, N., Nakanishi, H., et al. 2007, PASJ, 59, 117

Lara-López, M. A., Bongiovanni, A., Cepa, J., et al. 2010, A\&A, 519, A31

Lequeux, J., Le Bourlot, J., Pineau des Forets, G., et al. 1994, A\&A, 292, 371

Leroy, A., Bolatto, A. D., Simon, J. D., \& Blitz, L. 2005, ApJ, 625, 763

Leroy, A. K., Walter, F., Brinks, E., et al. 2008, AJ, 136, 2782

Leroy, A. K., Bolatto, A., Bot, C., et al. 2009a, ApJ, 702, 352

Leroy, A. K., Walter, F., Bigiel, F., et al. 2009b, AJ, 137, 4670

Leroy, A. K., Bolatto, A., Gordon, K., et al. 2011, ApJ, 737, 12

Leroy, A. K., Walter, F., Sandstrom, K., et al. 2013, AJ, 146, 19

Lisenfeld, U., Espada, D., Verdes-Montenegro, L., et al. 2011, A\&A, 534, A102

Lisker, T., Glatt, K., Westera, P., \& Grebel, E. K. 2006, AJ, 132, 2432

Lombardi, M., Alves, J., \& Lada, C. J. 2006, A\&A, 454, 781

Loose, H.-H., \& Thuan, T. X. 1986, in Star-forming Dwarf Galaxies and Related Objects, eds. D. Kunth, T. X. Thuan, J. Tran Thanh Van, J. Lequeux, \& J. Audouze (Éditions Frontières), 73

Lu, Z., Mo, H. J., \& Lu, Y. 2015, MNRAS, 450, 606

Madden, S. C., Poglitsch, A., Geis, N., Stacey, G. J., \& Townes, C. H. 1997, ApJ, 483, 200

Meyer, H. T., Lisker, T., Janz, J., \& Papaderos, P. 2014, A\&A, 562, A49

Mizuno, N., Rubio, M., Mizuno, A., et al. 2001, PASJ, 53, L45

Pettini, M., \& Pagel, B. E. J. 2004, MNRAS, 348, L59

Pety, J. 2005, in SF2A-2005: Semaine de l'Astrophysique Française, eds. F. Casoli, T. Contini, J. M. Hameury, \& L. Pagani, 721

Poglitsch, A., Krabbe, A., Madden, S. C., et al. 1995, ApJ, 454, 293

Rémy-Ruyer, A., Madden, S. C., Galliano, F., et al. 2014, A\&A, 563, A31

Saintonge, A. 2007, AJ, 133, 2087

Saintonge, A., Kauffmann, G., Kramer, C., et al. 2011a, MNRAS, 415, 32

Saintonge, A., Kauffmann, G., Wang, J., et al. 2011b, MNRAS, 415, 61

Sandstrom, K. M., Leroy, A. K., Walter, F., et al. 2013, ApJ, 777, 5

Schmidt, M. 1959, ApJ, 129, 243

Schruba, A., Leroy, A. K., Walter, F., et al. 2011, AJ, 142, 37

Schruba, A., Leroy, A. K., Walter, F., et al. 2012, AJ, 143, 138

Shetty, R., Glover, S. C., Dullemond, C. P., et al. 2011, MNRAS, 415, 3253

Solomon, P. M., \& Vanden Bout, P. A. 2005, ARA\&A, 43, 677

Springob, C. M., Haynes, M. P., Giovanelli, R., \& Kent, B. R. 2005, ApJS, 160, 149

Swaters, R. A., van Albada, T. S., van der Hulst, J. M., \& Sancisi, R. 2002, A\&A, 390,829

Tacconi, L. J., Genzel, R., Smail, I., et al. 2008, ApJ, 680, 246

Taylor, C. L., Kobulnicky, H. A., \& Skillman, E. D. 1998, AJ, 116, 2746

Toloba, E., Boselli, A., Gorgas, J., et al. 2009, ApJ, 707, L17

van der Marel, R. P., Alves, D. R., Hardy, E., \& Suntzeff, N. B. 2002, AJ, 124, 2639

van Zee, L., Salzer, J. J., \& Skillman, E. D. 2001, AJ, 122, 121

Wen, X.-Q., Wu, H., Zhu, Y.-N., et al. 2013, MNRAS, 433, 2946

Wen, X.-Q., Wu, H., Zhu, Y.-N., et al. 2014, MNRAS, 438, 97

Williams, M. J., Bureau, M., \& Cappellari, M. 2010, MNRAS, 409, 1330

Wilson, C. D. 1995, ApJ, 448, L97

Wolfire, M. G., Hollenbach, D., \& McKee, C. F. 2010, ApJ, 716, 1191

Wong, T., \& Blitz, L. 2002, ApJ, 569, 157

Wright, E. L., Eisenhardt, P. R. M., Mainzer, A. K., et al. 2010, AJ, 140, 1868

York, D. G., Adelman, J., Anderson, Jr., J. E., et al. 2000, AJ, 120, 1579

Young, J. S., \& Scoville, N. Z. 1991, ARA\&A, 29, 581

Zhukovska, S. 2014, A\&A, 562, A76

Zibetti, S., Charlot, S., \& Rix, H.-W. 2009, MNRAS, 400, 1181 
M. Grossi et al.: Star-forming dwarf galaxies in the Virgo cluster: the link between molecular gas, atomic gas, and dust

\section{Appendix A: Supplementary tables}

Table A.1. Pointing list, total integration time (ON+OFF), and Herschel/SPIRE photometry at $250 \mu \mathrm{m}$ in a circular aperture of radius $r=18^{\prime \prime} 6$.

\begin{tabular}{|c|c|c|c|c|}
\hline ID & $\begin{array}{c}\mathrm{RA} \\
{[\mathrm{J} 200]}\end{array}$ & $\begin{array}{c}\text { Dec } \\
{[\mathrm{J} 200]}\end{array}$ & $\begin{array}{l}\text { Time } \\
{[\mathrm{min}]}\end{array}$ & $\begin{array}{l}S_{250}^{\text {beam }} \\
{[\mathrm{mJy}]}\end{array}$ \\
\hline VCC10 & $12: 09: 24.90$ & $13: 34: 28.0$ & 30 & $189.0 \pm 8.8$ \\
\hline VCC87 & $12: 13: 41.30$ & $15: 27: 13.0$ & 65 & $105.3 \pm 5.2$ \\
\hline VCC 135 & $12: 15: 06.70$ & $12: 01: 00.0$ & 15 & $239.3 \pm 11.0$ \\
\hline VCC144 & $12: 15: 18.30$ & $05: 45: 39.0$ & 30 & $154.1 \pm 7.5$ \\
\hline VCC172 & $12: 16: 00.40$ & 04:39:03.0 & 15 & $66.6 \pm 4.6$ \\
\hline VCC213(a) & $12: 16: 56.00$ & $13: 37: 31.5$ & 20 & $391.3 \pm 15.5$ \\
\hline VCC213(b) & $12: 16: 56.73$ & $13: 37: 25.8$ & 20 & $277.7 \pm 9.9$ \\
\hline VCC324 & 12:19:09.90 & $03: 51: 21.0$ & 30 & $220.8 \pm 9.2$ \\
\hline VCC334 & $12: 19: 14.20$ & $13: 52: 56.0$ & 130 & $51.1 \pm 3.9$ \\
\hline VCC 340 & $12: 19: 22.10$ & $05: 54: 38.0$ & 30 & $152.5 \pm 6.9$ \\
\hline VCC562 & $12: 22: 35.90$ & $12: 09: 27.0$ & 40 & $53.2 \pm 3.5$ \\
\hline VCC693 & $12: 24: 03.35$ & $05: 10: 50.4$ & 30 & $78.7 \pm 4.5$ \\
\hline VCC699(a) & $12: 24: 07.50$ & $06: 36: 27.8$ & 30 & $363.0 \pm 12.6$ \\
\hline VCC699(b) & $12: 24: 08.14$ & $06: 36: 31.0$ & 30 & $343.8 \pm 12.1$ \\
\hline VCC737 & $12: 24: 39.40$ & $03: 59: 44.0$ & 55 & $90.8 \pm 4.6$ \\
\hline VCC841 & $12: 25: 47.40$ & $14: 57: 07.5$ & 50 & $94.2 \pm 6.0$ \\
\hline VCC 1437 & $12: 32: 33.50$ & $09: 10: 25.0$ & 20 & $134.2 \pm 6.7$ \\
\hline VCC1575(a) & $12: 34: 39.96$ & 07:09:52.0 & 10 & $467.4 \pm 17.2$ \\
\hline VCC1575(b) & $12: 34: 39.65$ & $07: 09: 41.8$ & 10 & $580.2 \pm 20.1$ \\
\hline VCC1575(c) & $12: 34: 39.28$ & 07:09:32.2 & 10 & $638.0 \pm 21.0$ \\
\hline VCC1575(d) & $12: 34: 38.80$ & 07:09:23.8 & 10 & $527.2 \pm 19.0$ \\
\hline VCC1575(e) & $12: 34: 40.06$ & 07:10:02.8 & 10 & $270.0 \pm 8.2$ \\
\hline VCC1686(a) & $12: 36: 44.26$ & $13: 15: 27.1$ & 30 & $434.8 \pm 15.4$ \\
\hline VCC1686(b) & $12: 36: 43.73$ & $13: 15: 13.2$ & 10 & $226.3 \pm 8.1$ \\
\hline VCC1686(c) & $12: 36: 43.73$ & $13: 15: 02.2$ & 10 & $338.8 \pm 11.5$ \\
\hline VCC1699 & $12: 37: 02.60$ & $06: 55: 31.0$ & 70 & $126.3 \pm 6.8$ \\
\hline VCC1725 & $12: 37: 40.57$ & $08: 33: 37.4$ & 30 & $139.4 \pm 5.9$ \\
\hline VCC1791 & $12: 39: 25.87$ & $07: 58: 04.6$ & 30 & $98.0 \pm 6.2$ \\
\hline
\end{tabular}

Table A.2. Aperture corrections calculated using the methods of Saintonge et al. (2011a) and Lisenfeld et al. (2011), source sizes, and correction factors for the finite angular sizes of the source.

\begin{tabular}{lcccccc}
\hline \hline ID & $f_{\text {ap }}^{S 11}$ & $f_{\text {ap }}^{L 11}$ & $\begin{array}{c}2 R_{\mathrm{u}} \\
{\left[{ }^{\prime \prime}\right]}\end{array}$ & $\begin{array}{c}\theta_{\mathrm{g}} \\
{\left[{ }^{\prime \prime}\right]}\end{array}$ & $f_{\mathrm{s}}^{\mathrm{u}}$ & $f_{\mathrm{s}}^{\mathrm{g}}$ \\
\hline VCC10 & 1.48 & $1.46 \pm 0.44$ & $<20$ & $<19$ & $1.07_{0.07}^{0.24}$ & $1.19_{0.19}^{0.56}$ \\
VCC87 & 1.82 & $2.04 \pm 0.61$ & - & - & - & - \\
VCC135 & 1.58 & $1.72 \pm 0.52$ & $3.7_{3.7}^{6.1}$ & $2.6_{2.6}^{4.9}$ & $1.01_{0.01}^{0.06}$ & $1.01_{0.01}^{0.10}$ \\
VCC144 & 1.22 & $1.26 \pm 0.38$ & $<13$ & $<10.4$ & $1.03_{0.03}^{0.10}$ & $1.06_{0.06}^{0.17}$ \\
VCC172 & 1.66 & $1.78 \pm 0.53$ & - & - & - & - \\
VCC213 & 1.41 & $1.65 \pm 0.49$ & ext & ext & ext & ext \\
VCC324 & 1.73 & $2.32 \pm 0.70$ & $18.9_{6.2}^{7.4}$ & $18.0_{7.7}^{14.4}$ & $1.28_{0.16}^{0.30}$ & $1.67_{0.45}^{0.33}$ \\
VCC334 & 1.18 & $1.30 \pm 0.39$ & - & - & - & - \\
VCC340 & 1.53 & $1.59 \pm 0.48$ & $17.7_{6.4}^{6.9}$ & $16.3_{7.4}^{12.1}$ & $1.24_{0.15}^{0.25}$ & $1.55_{0.38}^{0.45}$ \\
VCC562 & 1.22 & $1.33 \pm 0.40$ & - & - & - & - \\
VCC693 & 1.58 & $2.03 \pm 0.61$ & - & - & - & - \\
VCC699 & 3.12 & $3.11 \pm 0.93$ & ext & ext & ext & ext \\
VCC737 & 1.51 & $1.53 \pm 0.46$ & - & - & - & - \\
VCC841 & 1.35 & $1.37 \pm 0.41$ & - & - & - & - \\
VCC1437 & 1.20 & $1.29 \pm 0.39$ & $12.5_{2.8}^{2.5}$ & $10.1_{2.7}^{2.8}$ & $1.12_{0.05}^{0.05}$ & $1.21_{0.10}^{0.13}$ \\
VCC1575 & 3.54 & $3.20 \pm 0.96$ & ext & ext & ext & ext \\
VCC1686 & 3.17 & $4.45 \pm 1.33$ & ext & ext & ext & ext \\
VCC1699 & 1.91 & $2.21 \pm 0.66$ & - & - & - & - \\
VCC1725 & 1.91 & $2.33 \pm 0.70$ & ext & ext & ext & ext \\
VCC1791 & 1.68 & $1.86 \pm 0.56$ & - & - & - & - \\
\hline
\end{tabular}

Table A.3. Stellar masses used to compare Virgo SFDs to the HRS sample, and $H$-band photometry.

\begin{tabular}{lcc}
\hline \hline ID & $\begin{array}{c}\log \left(M_{*}\right) \\
{\left[M_{\odot}\right]}\end{array}$ & $\begin{array}{c}m_{\mathrm{H}} \\
{[\mathrm{mag}]}\end{array}$ \\
\hline VCC10 & 8.67 & 12.81 \\
VCC87 & 8.09 & 13.16 \\
VCC135 & 9.40 & 11.48 \\
VCC144 & 8.17 & 13.04 \\
VCC172 & 8.55 & 12.62 \\
VCC213 & 8.75 & 11.48 \\
VCC324 & 8.35 & 11.98 \\
VCC334 & 7.77 & 13.28 \\
VCC340 & 8.82 & 12.29 \\
VCC562 & 7.37 & 15.70 \\
VCC693 & 8.21 & - \\
VCC699 & 8.91 & 11.28 \\
VCC737 & 8.10 & 12.73 \\
VCC841 & 8.35 & 13.16 \\
VCC1437 & 8.43 & 12.32 \\
VCC1575 & 9.19 & 10.76 \\
VCC1686 & 8.82 & 10.99 \\
VCC1699 & 8.12 & 12.37 \\
VCC1725 & 8.42 & 12.46 \\
VCC1791 & 8.15 & 12.81 \\
\hline
\end{tabular}




\section{Appendix B: $\mathrm{CO}(1-0)$ and $\mathrm{CO}(2-1)$ spectra of Virgo SFDs}
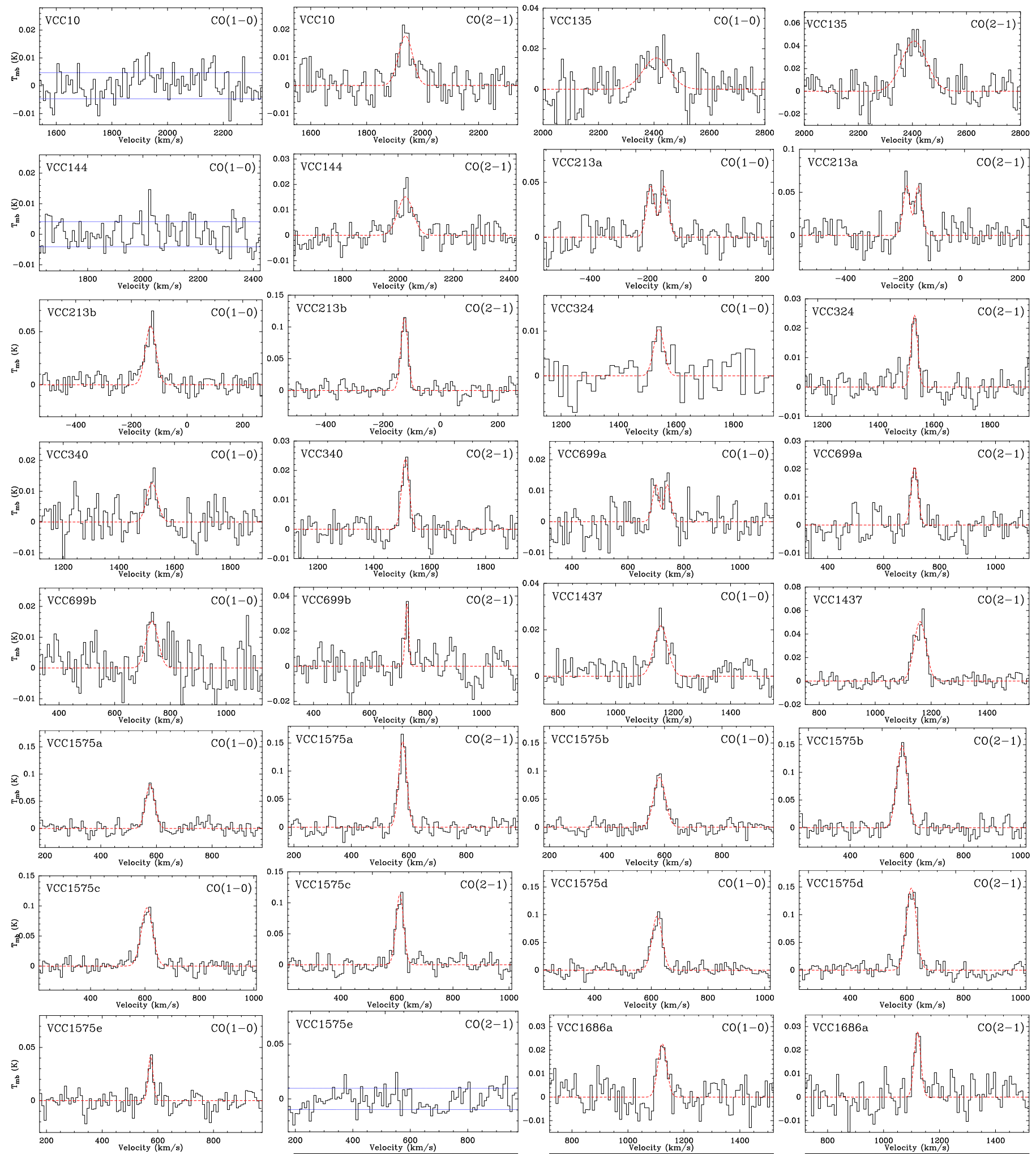

$=\underbrace{600}_{\mathbf{V e l o c i t y ~}(\mathrm{km} / \mathrm{s})}$
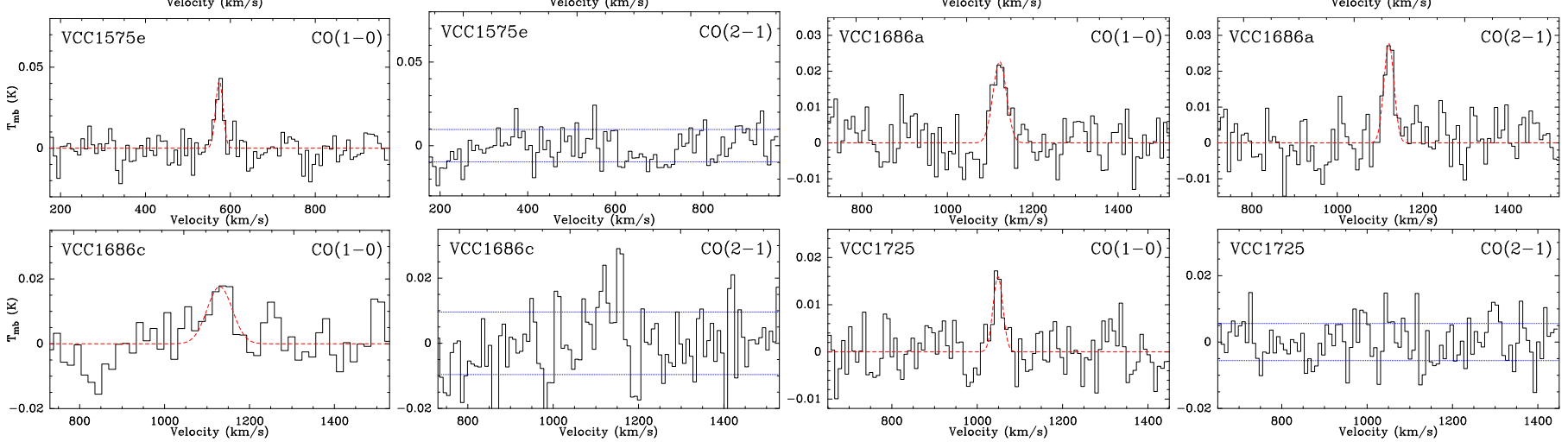

Fig. B.1. $\mathrm{CO}(1-0)$ and $\mathrm{CO}(2-0)$ spectra of galaxies detected in at least one band. The dashed curves show the best fit to the lines. The dotted horizontal lines indicate the $\pm 1 \sigma \mathrm{rms}$ level in case of a non-detection. 
M. Grossi et al.: Star-forming dwarf galaxies in the Virgo cluster: the link between molecular gas, atomic gas, and dust
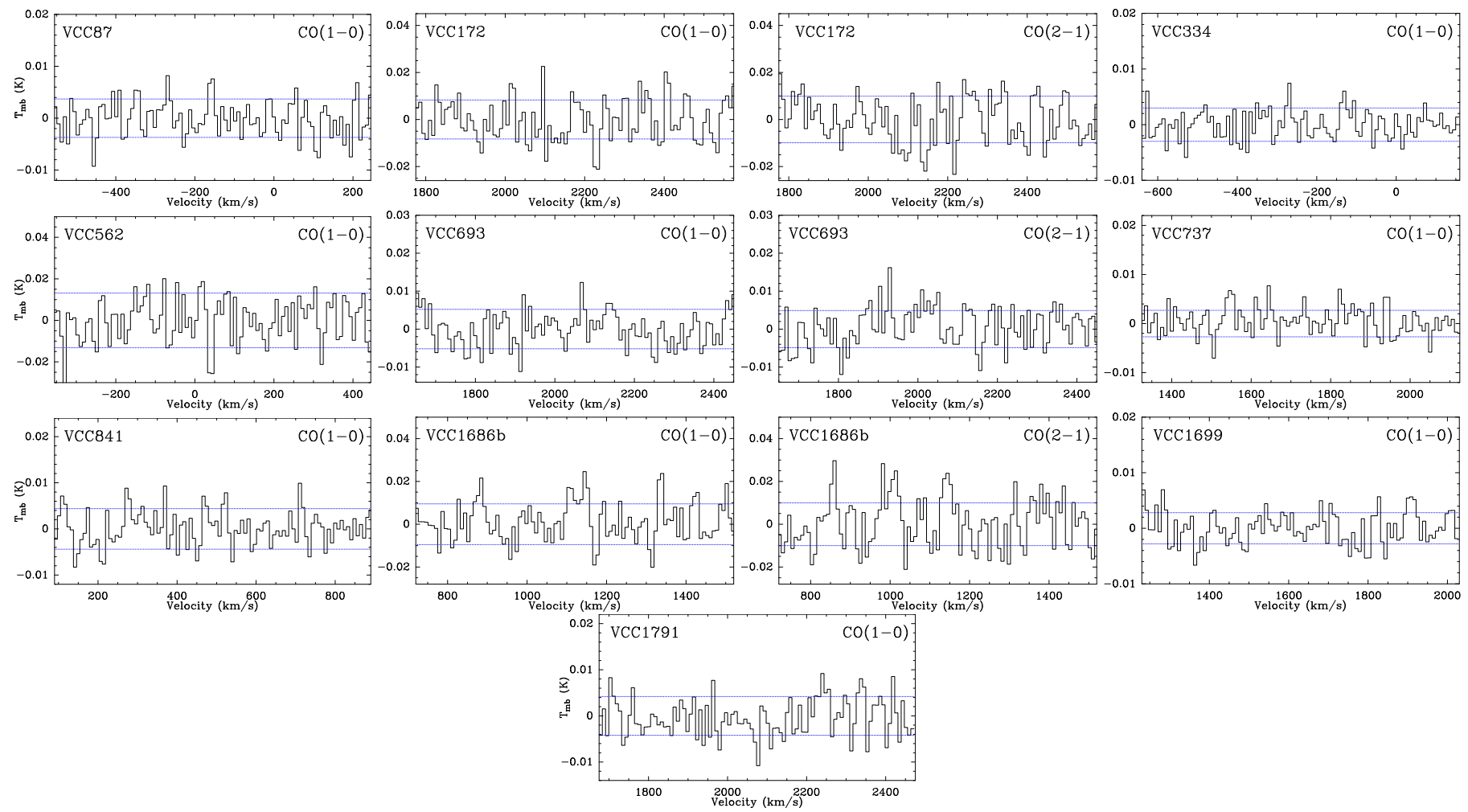

Fig. B.2. $\mathrm{CO}(1-0)$ and $\mathrm{CO}(2-1)$ spectra of non-detections. The dotted horizontal lines show the $\pm 1 \sigma \mathrm{rms}$ level. 